ARTICLE

DOI: $10.1038 / \mathrm{s} 41467-018-06451-3$

\title{
RIP2 filament formation is required for NOD2 dependent NF-kB signalling
}

Erika Pellegrini ${ }^{1}$, Ambroise Desfosses ${ }^{2}$, Arndt Wallmann ${ }^{3}$, Wiebke Manuela Schulze ${ }^{1}$, Kristina Rehbein ${ }^{3}$, Philippe Mas², Luca Signor (10 4 , Stephanie Gaudon', Grasilda Zenkeviciute ${ }^{1,6}$, Michael Hons ${ }^{1}$, Helene Malet ${ }^{2}$, Irina Gutsche ${ }^{2}$, Carsten Sachse (10 5, Guy Schoehn (10 2, Hartmut Oschkinat ${ }^{3}$ \& Stephen Cusack (D) ${ }^{1}$

Activation of the innate immune pattern recognition receptor NOD2 by the bacterial muramyl-dipeptide peptidoglycan fragment triggers recruitment of the downstream adaptor kinase RIP2, eventually leading to NF- $\mathrm{B}$ B activation and proinflammatory cytokine production. Here we show that full-length RIP2 can form long filaments mediated by its caspase recruitment domain (CARD), in common with other innate immune adaptor proteins. We further show that the NOD2 tandem CARDs bind to one end of the RIP2 CARD filament, suggesting a mechanism for polar filament nucleation by activated NOD2. We combine X-ray crystallography, solid-state NMR and high-resolution cryo-electron microscopy to determine the atomic structure of the helical RIP2 CARD filament, which reveals the intermolecular interactions that stabilize the assembly. Using structure-guided mutagenesis, we demon-

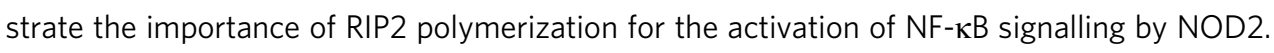
Our results could be of use to develop new pharmacological strategies to treat inflammatory diseases characterised by aberrant NOD2 signalling.

\footnotetext{
${ }^{1}$ European Molecular Biology Laboratory, 71 Avenue des Martyrs, CS 90181, 38042 Grenoble, Cedex 9, France. ${ }^{2}$ Univ. Grenoble Alpes, CNRS, CEA, CNRS, IBS, F-38000 Grenoble, France. ${ }^{3}$ Leibniz-Forschungsinstitut für Molekulare Pharmakologie (FMP), Department for NMR-supported Structural, Biology Robert-Rössle-Straße 10, 13125 Berlin, Germany. ${ }^{4}$ University Grenoble Alpes, CEA, CNRS, IBS, F-38000 Grenoble, France. ${ }^{5}$ European Molecular Biology Laboratory, Structural and Computational Biology Unit, Meyerhofstraße 1, 69117 Heidelberg, Germany. ${ }^{6}$ Present address: Grasilda Zenkeviciute, Department of Pharmacology, University of Cambridge, Tennis Court Road, Cambridge CB2 1PD, United Kingdom. Correspondence and requests for materials should be addressed to S.C. (email: cusack@embl.fr)
} 
T o respond rapidly to microbial infection, the innate immune system uses pattern recognition receptors (PRRs) to detect pathogen-specific molecules called pathogenassociated molecular patterns (PAMPs) ${ }^{1}$. This primary event leads to receptor activation and signalling via an immediate downstream adaptor protein. Here we focus on the cytosolic PRR NOD2 (nucleotide oligomerization domain 2) ${ }^{2}$ (\#285), which senses bacterial infection by recognizing the peptidoglycan breakdown product MDP (muramyl di-peptide) ${ }^{3,4}$, and the adaptor protein RIP2 (receptor-interacting protein 2, also known as RICK ${ }^{5}$.

NOD2 belongs to the Nod-like receptor (NLR) family, which are characterised by three functional domains: a C-terminal ligand-binding domain comprising leucine-rich repeats (LRRs), a central ATP-binding and oligomerization domain (nucleotide oligomerization domain, NOD) and an N-terminal effector death-domain (DD), which in the case of NOD2 is a double CARD (caspase recruitment domain) ${ }^{6,7}$. The downstream adaptor RIP2 belongs to the RIP kinase family and comprises an Nterminal kinase domain, a C-terminal CARD domain and a bridging intermediate domain ${ }^{8}$. Upon cognate ligand binding, NOD2 oligomerizes and recruits RIP2 via CARD-CARD interactions $5,7,9-14$. After RIP2 auto-phosphorylation and ubiquitination, RIP2 becomes a platform for downstream protein effectors including several ubiquitin E3-ligases ${ }^{15-17}$. Eventually, the NOD2 signalling pathway triggers an inflammatory response through NF-KB, MAPK activation and autophagy as well as the production of anti-bacterial peptides, which protect gut epithelial cells from both residual flora and pathogen invasion $78,18,19$.

Excessive or absent NOD2-RIP2 signalling is associated with several genetic and non-genetic inflammatory diseases, which lack specific and effective therapies. Loss-of-function single nucleotide polymorphisms (SNPs) in NOD2, which result in impaired epithelial mucosal barrier function, are one of the major genetic susceptibility factors for Crohn's disease (CD), an increasingly frequent disorder in the western world ${ }^{20-23}$. On the other hand, gain-of-function SNPs of NOD2 can cause Blau syndrome and early-onset sarcoidosis (EOS), which are systematic granulomatous inflammatory diseases ${ }^{24-27}$. Aberrant overactive NOD2-RIP2 signalling might also be involved in inflammatory arthritis, asthma, colorectal cancer and multiple sclerosis, as suggested by animal models and association studies 9 Despite the importance of the NOD2 signalling pathway in health and disease, there is still an incomplete understanding of its molecular basis. In particular, obtaining insight into the mechanism by which the ligand-induced oligomerization of NOD2 induces RIP2 activation is an important goal since it could lead to the development of new therapies for these clinical conditions.

Recent studies of other intracellular innate immune signalling pathways have shown that ligand-induced PRR oligomerization promotes the polymerization of the adaptor protein through their $\mathrm{DDs}^{28,29}$. This interaction leads to the formation of fibrillar protein assemblies, called signalosomes, which link the upstream danger signal to the downstream enzyme-driven pathway. Examples are the anti-viral pathway mediated by RIG-I and downstream adaptor MAVS ${ }^{30-32}$, the inflammasome pathway involving NLRP3, ASC and caspase-133,34, and the recently described T-cell/B-cell signalosome CARMA1-BLC10-MALT1 ${ }^{35}$. Several groups have investigated the interaction between NOD2CARDS and RIP2CARD ${ }^{12,14}$, but the question remains whether this hetero-CARD interaction also leads to formation of a higher-order signalosome?

Here we present biophysical and structural data, showing that full-length RIP2 can form filaments that are mediated by RIP2CARD oligomerization. We report the atomic structure of
RIP2CARD filaments, solved by high-resolution cryo-electron microscopy (cryo-EM), which reveals the molecular interactions underlying its assembly. We show that NOD2CARDS can bind to one end of the RIP2CARD filaments, suggesting that NOD2 activation could nucleate RIP2 filament formation. Consistent with this, we use structure-guided mutants, designed to specifically disrupt RIP2 filament formation, to demonstrate, in vitro and in cell based assays, the relevance of RIP2 polymerization for the activation of NF-kB signalling following NOD2 stimulation.

\section{Results}

RIP2 forms filaments in vitro via its CARD domain. Using the baculovirus system in $s f 21$ cells, we expressed and purified recombinant full-length human RIP2 with a cleavable maltosebinding protein (MBP) tag at the N-terminus ${ }^{36}$ (MBP-RIP2fl) (Fig. 1a-c). Negative-stain EM of MBP-RIP2fl eluting from the amylose resin shows that the sample is a mixture of aggregates and oligomeric protein (Supplementary Fig. 1a). Addition of ATP and magnesium promotes elongation of the aggregates into a filamentous structure (Supplementary Fig. 1b). After MBP tag cleavage, the protein was further purified by size exclusion chromatography yielding a void volume (VV) fraction and a soluble, non-aggregated fraction denoted RIP2fl (Fig. 1b-c, Supplementary Fig. 2). Analysis of the non-aggregated RIP2fl by electrospray ionization (ESI) mass spectrometry confirmed that the protein is highly phosphorylated (Supplementary Fig. 1c) and an in vitro phosphorylation assay showed that it is capable of further self-phosphorylation and is thus functionally active (Fig. 1d). Negative-stain EM of the VV fraction shows irregular aggregates with some short filaments displaying a central filamentous core (Fig. 1e, Supplementary Fig. 1d-e), whereas imaging the RIP2fl fraction confirms that it is non-aggregated and presumably dimeric (Fig. 1c, f and Supplementary Fig. 2). When ATP and magnesium were added to the non-aggregated RIP2fl fraction, the protein oligomerized into long filaments of diameter $30-40 \mathrm{~nm}$ and variable length $(0.1-1 \mu \mathrm{m})$ and which have a tendency to side-by-side aggregation (Fig. 1g). Extended filaments could also be obtained from the VV fraction by adding nonaggregated RIP2fl, ATP and magnesium, with the VV aggregates acting as seeds (Supplementary Fig. 1f).

We then investigated the importance of different nucleotides in promoting RIP2fl polymerization. For this, we used the uncleaved MBP-RIP2fl fusion protein (Fig. 2a) rather than tag-free RIP2fl. This is because when ATP and magnesium are added, MBPRIP2fl polymerizes into short filaments $(0.1-0.2 \mu \mathrm{m})$ that aggregate less compared to those made with tag-free protein, making them easier to visualise by negative-stain EM (Fig. 2b). MBP-RIP2fl polymerization was imaged in the presence of magnesium alone (Fig. 2c) or together with either ATP (Fig. 2b), AMP (Fig. 2d), ADP (Fig. 2e) or the ATP analogue AMPPCP (Fig. 2f). The micrographs reveal that MBP-RIP2fl begins to polymerize in the presence of magnesium and the filaments elongate when any adenosine nucleotide is provided. Given that the recombinant RIP2fl is active and already highly phosphorylated (Fig. 1d and Supplementary Fig. 1c), these data suggest that ATP (or other adenosine nucleotide) boosts RIP2fl polymerization by stabilizing the kinase domain in a pro-filament conformation, rather than by promoting further autophosphorylation.

Interestingly, the polymerized RIP2fl filaments appear to have an inner core decorated with surface projections (Fig. 1g, Supplementary Fig. 1e). In analogy to other adaptor proteins capable of polymerization via their $\mathrm{DDs}^{30,32-34,37}$, we hypothesized that the RIP2CARD forms the filament core, whilst the outer projections correspond to the kinase domain. Therefore, we 

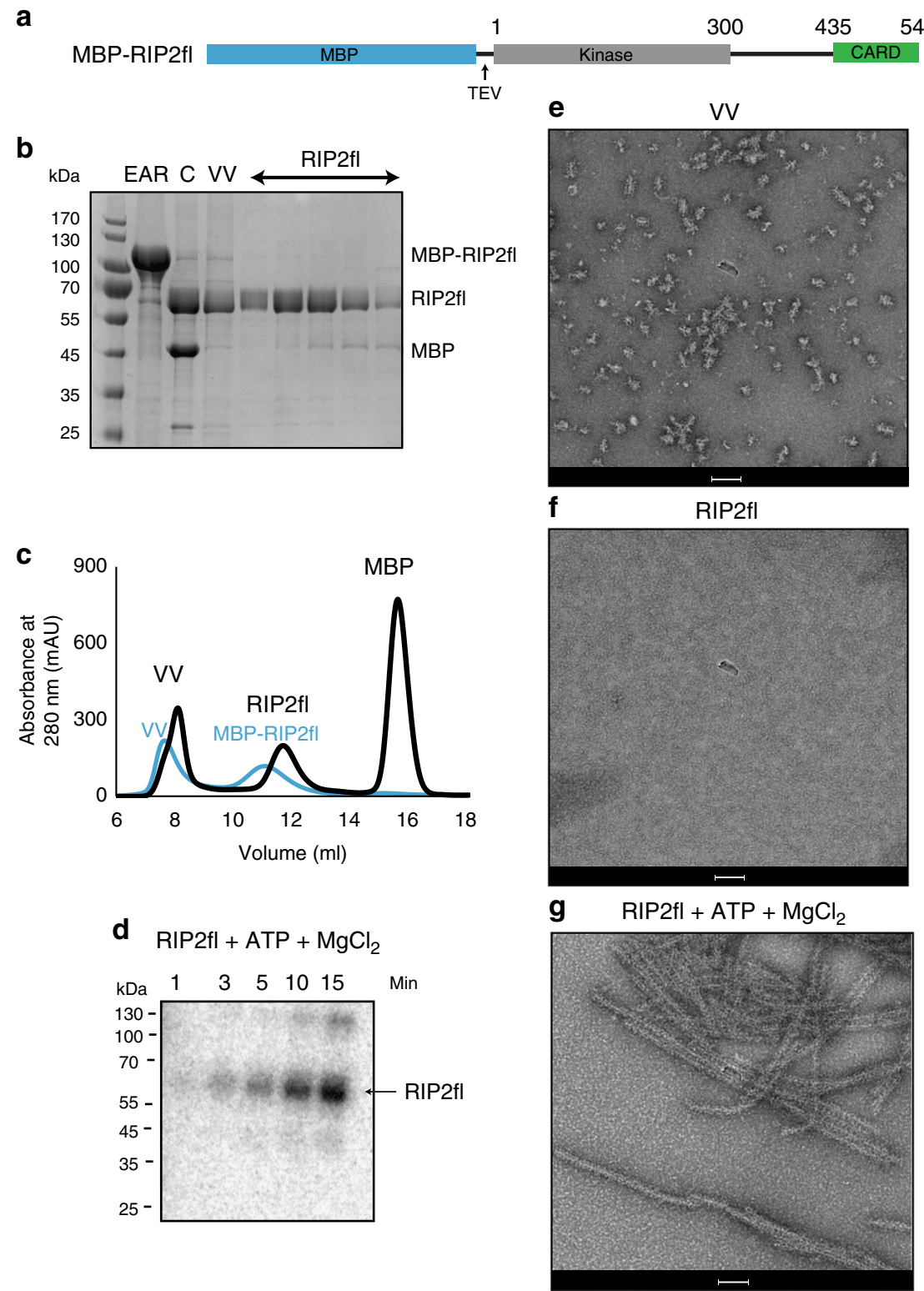

Fig. 1 Full-length RIP2 forms filaments in vitro. a Domain organization of the MBP-RIP2fl construct used for expression and purification of recombinant RIP2fl from sf21 insect cells. b $12.5 \%$ SDS-PAGE showing recombinant RIP2fl at consecutive purifications steps. c Typical size exclusion chromatography profile for tagged (blue) and tag-free RIP2fl (black). $\mathbf{d}$ In vitro kinase activity of RIP2fl. Complete auto-phosphorylation is achieved after 15 min. e-g Negative-stain micrographs of (e) VV RIP2fl, (f) RIP2fl and (g) RIP2fl with added ATP and magnesium chloride. Scale bars are $100 \mathrm{~nm}$. EAR: eluate from amylose resin, C: sample after tag cleavage as loaded on size-exclusion chromatography, VV: size exclusion chromatography void volume, Min: minutes

expressed and purified from E. coli recombinant RIP2CARD (residues 435-540) with a cleavable MBP tag at the N-terminus (MBP-RIP2CARD, Fig. 3a-c). Upon MBP tag cleavage by the Tobacco Etch Virus (TEV) protease, RIP2CARD mainly migrated in the void volume ( $\mathrm{VV}$ ) of the size-exclusion chromatography column (Fig. 3b). Negative-stain micrographs revealed that RIP2CARD from the VV forms long filaments (Fig. 3d), which have similar length to the RIP2fl filaments, but a smaller diameter of less than $10 \mathrm{~nm}$.

The structure of monomeric RIP2CARD. To aid structural analysis of the RIP2CARD filaments, we determined the X-ray crystal structure of RIP2CARD (residues 435-540), using a construct with crystallisable $\mathrm{MBP}^{38}$ fused at the N-terminus (crystMBP-RIP2CARD, Fig. 3a, e). This construct crystallised in space group $P 2_{1}$ with four molecules per asymmetric unit (Supplementary Fig. 3). The structure was solved by molecular replacement using NLRP1 CARD domain with a crystallisable MBP at the N-terminus (PDB accession code 4IFP, ${ }^{39}$ ) as search model and refined at $3.3 \AA$ resolution (Supplementary Table 1). RIP2CARD has the typical CARD fold comprising a Greek key helical bundle with the $\mathrm{N}$ - and C-termini oriented in the same direction and with helix $\mathrm{H} 1$ broken into two shorter helices: H1a and H1b (Fig. 3e, Supplementary Fig. 3-4). The RIP2CARD crystal structure is very similar to the previously reported solution NMR structure (Fig. 3f, PDB code: $2 \mathrm{~N} 7 \mathrm{Z})^{40}$, with a root-meansquare deviation (RMSD) of all Ca positions of $0.95 \AA$. Interestingly $\mathrm{H} 6$ is absent in both the crystal and NMR structures and replaced by a long C-terminal loop, visible only in the NMR structure, which contains putative phosphorylation sites ${ }^{41-43}$. 
a

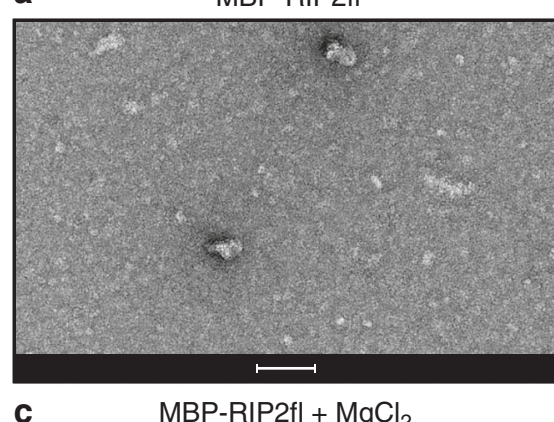

C

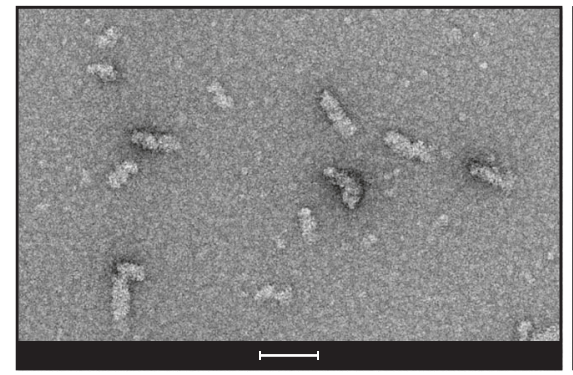

e

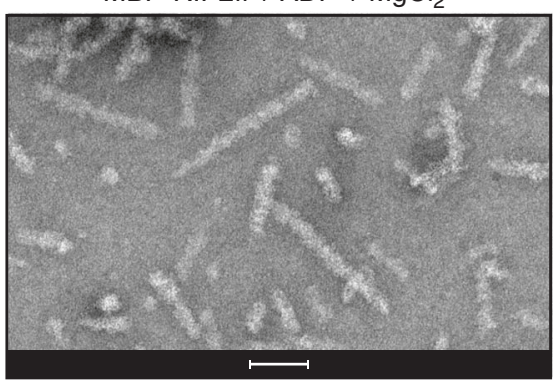

b $\quad$ MBP-RIP2fl $+\mathrm{ATP}+\mathrm{MgCl}_{2}$

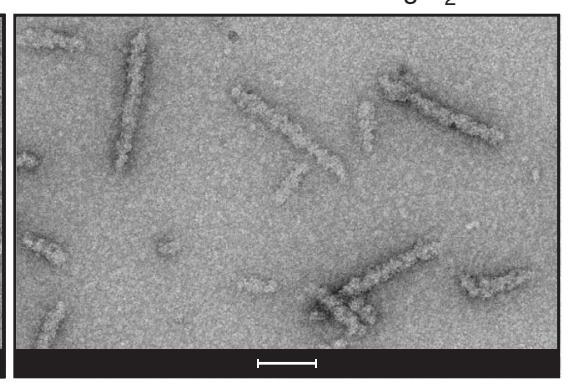

d $\quad$ MBP-RIP2fl $+\mathrm{AMP}+\mathrm{MgCl}_{2}$

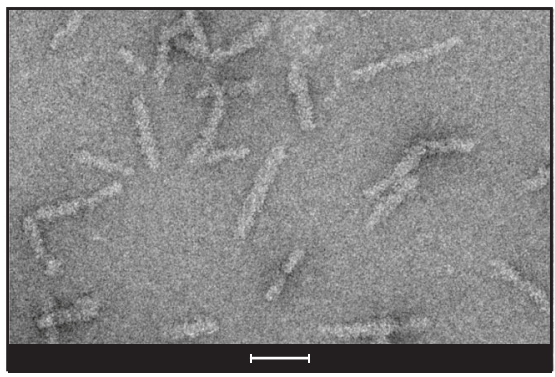

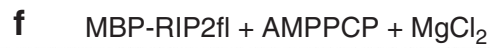

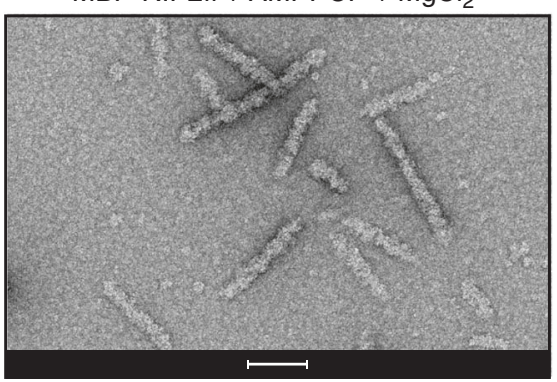

Fig. 2 Full-length RIP2 filaments are promoted by nucleotide binding. a-c Negative-stain micrographs of MBP-RIP2fl filaments obtained from (a) MBPRIP2fl alone, (b) MBP-RIP2fl plus ATP dissolved in magnesium chloride buffer, (c) MBP-RIP2fl plus magnesium, 5 mM nucleotide dissolved in buffer containing magnesium chloride: (d) AMP, (e) ADP and (f) AMPPCP. Scale bars are $100 \mathrm{~nm}$

The structure of RIP2CARD within filaments. We used solidstate NMR to study the structure of RIP2CARD within the filament. In order to obtain backbone resonance assignments, we recorded ${ }^{1} \mathrm{H}$-detected $(\mathrm{H}) \mathrm{CANH},(\mathrm{HCO}) \mathrm{CA}(\mathrm{CO}) \mathrm{NH}$, (HCA)CB (CA)NH, (HCA)CB(CACO)NH, (H)CONH and (H)CO(CA)NH spectra on ${ }^{2} \mathrm{H},{ }^{13} \mathrm{C},{ }^{15} \mathrm{~N}$-labeled and $100 \%$ back-exchanged RIP2CARD samples at $60 \mathrm{kHz}$ magic angle spinning (MAS) ${ }^{44}$. This data was evaluated together with ${ }^{13} \mathrm{C}$-detected ${ }^{13} \mathrm{C}-{ }^{13} \mathrm{C}$ DARR correlations on protonated samples, that were either uniformly ${ }^{13} \mathrm{C}$-labelled or selectively-labelled using $\left[2-{ }^{13} \mathrm{C}\right]-$ or $\left[1,3-{ }^{13} \mathrm{C}\right]$-glycerol as carbon source during protein expres$\operatorname{sion}^{45,46}$. The analysis of the ${ }^{1} \mathrm{H}$-detected data yielded the sequence specific assignment for residues Q441 to Q507 (Supplementary Table 2), except for the loop residues $448-451$ and 497. The chemical shifts of the assigned residues of filamentous RIP2CARD closely match many chemical shifts of monomeric RIP2CARD in solution showing that the overall conformation is maintained upon filament formation (Fig. $3 \mathrm{~g}$ ). We were not able to assign any cross-peaks to the $\mathrm{C}$-terminal 29 residues that were reported to be flexible by solution NMR investigations ${ }^{40}$. To check whether these signals are absent in our MAS NMR spectra, we inspected ${ }^{13} \mathrm{C}-{ }^{13} \mathrm{C}$ correlation spectra of the samples with a 2or 1,3-glycerol labelling pattern. At short mixing times, the amino acids Leu, Pro, Thr and Val lead to characteristic cross-peak pattern that allow for a counting of signals. We observed signals corresponding to 9 of 14 leucine residues, 6 of $6 \mathrm{Thr}, 5$ of $7 \mathrm{Val}$ and only 1 of 4 proline residues (Supplementary Fig. 5). Relying on the distribution of the respective amino acid types in the sequence, this strongly suggests that the missing signals concern residues in the C-terminal segment from 512 to 540 . Especially the absence of three proline signal sets, only one being detected, indicates strong structural heterogeneity or mobility in that region where they cluster. Furthermore, the number of missing Leu and Val signal sets corresponds to the number present in the C-terminus and thus corroborates the lack of an ordered structure there, indicating that $\mathrm{H} 6$ is also absent in filamentous RIP2CARD.

NOD2CARDS bind to one end of the RIP2CARD filament. We have shown that both RIP2fl and RIP2CARD samples form filaments in vitro. However, in the cellular context, we expect that such polymerization is initiated by NOD2 oligomerization in response to cognate ligand binding. To recapitulate the core elements of this process, we set out to reconstitute in vitro a filamentous sample comprising the CARDS of both proteins that would be suitable for high-resolution structure determination by cryo-EM.

We first investigated whether NOD2 could be detected by immuno-gold labelling in RIP2CARD filaments formed in the presence of NOD2, following what was previously done for both 
a

MBP-RIP2CARD

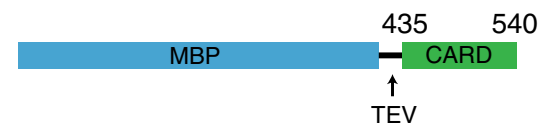

crystMBP-RIP2CARD

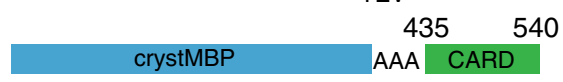

b

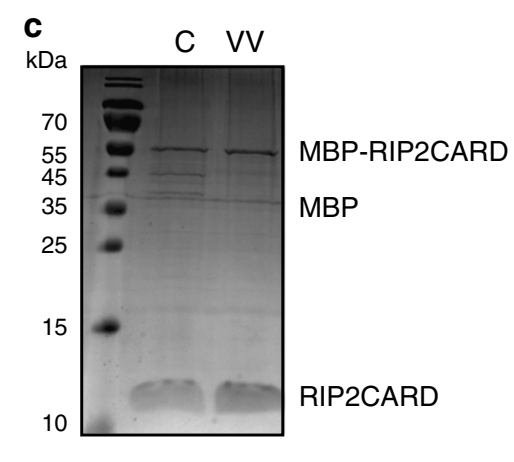

d RIP2CARD
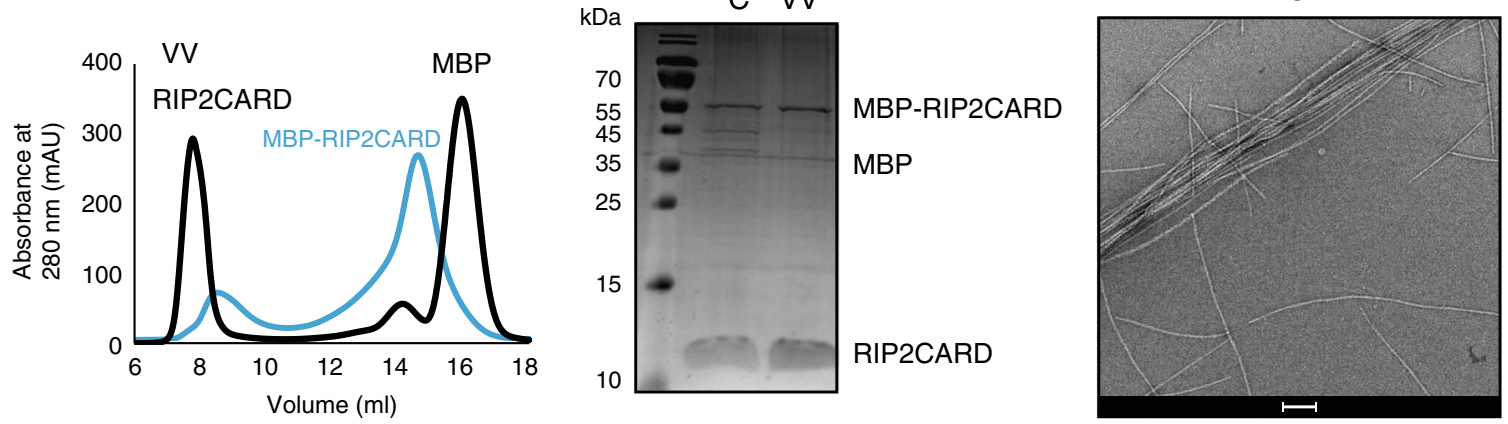

e

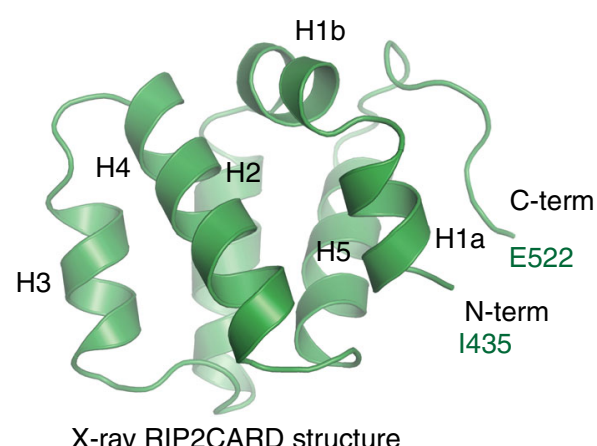

$\mathbf{f}$

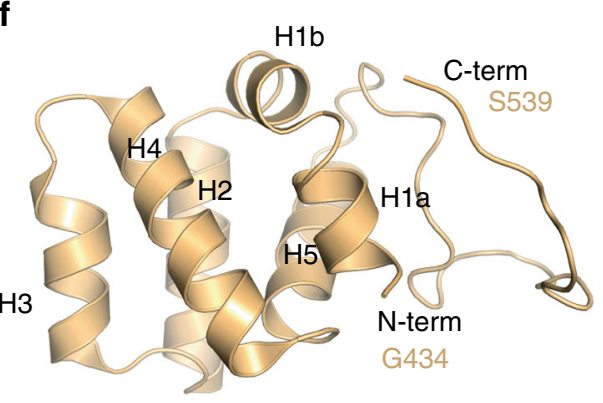

NMR RIP2CARD structure

$\mathbf{g}$ Solid state NMR on filamentous RIP2CARD

Solution state NMR on monomeric RIP2CARD

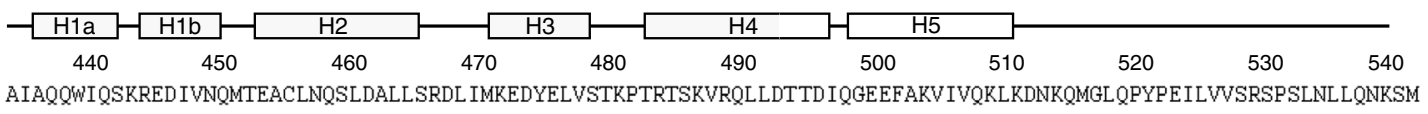

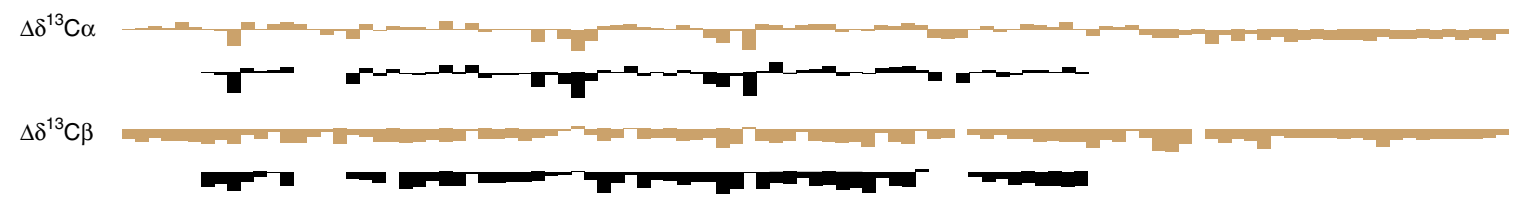

Fig. 3 Structure of monomeric RIP2CARD. a Domain organization of MBP-RIP2CARD constructs used for expression and purification of recombinant RIP2CARD from E. coli Rosetta 2. MBP-RIP2CARD was used for characterising the polymerization ability of the CARD domain and for NMR experiments. CrystMBP-RIP2CARD was used for crystallization. b Typical size exclusion chromatography profiles of RIP2CARD purification showing both tagged (blue) and tag-free RIP2CARD (black). c $17 \%$ SDS-PAGE showing typical sample obtained from RIP2CARD purification. The SDS-PAGE indicates that tag cleavage by TEV is incomplete. d Negative-stain electron micrograph of RIP2CARD VV. Scale bar is $100 \mathrm{~nm}$. e Ribbon diagram of the RIP2CARD crystal structure reported in this paper $\mathbf{f}$ Ribbon diagram of the solution NMR structure of RIP2CARD (PDB: 2N7Z). $\mathbf{g}$ Comparison of secondary chemical shifts of RIP2CARD as a monomer in solution (yellow; BMRB entry: 25828) and in the filament, determined by proton-detected solid-state NMR (black). Experimental ${ }^{13} \mathrm{C} \alpha$ and ${ }^{13} \mathrm{C} \beta$ chemical shifts were subtracted from the respective random coil values for each amino acid type $(\Delta \delta \mathrm{C} \alpha, \Delta \delta C \beta)^{79,80}$. C: sample after tag cleavage as loaded on size-exclusion chromatography, VV: size exclusion chromatography void volume

the AIM2-ASC or NLRP3-ASC complexes ${ }^{33}$. Using the baculovirus insect cell system we expressed and purified a truncated form of NOD2 with a TEV cleavable MBP tag, comprising the CARDS and NOD, but lacking the LRR domain (MBPNOD2 $\triangle$ LRR, residues 1-619) (Fig. 4a). This construct is presumed to be derepressed with the CARDS available for interaction. Indeed, a similar NLRP3 construct proved to be a more powerful ASC polymerization promoter compared with the full-length receptor ${ }^{33}$.

Purified and tag-free NOD2 $\triangle \mathrm{LRR}$ eluted mainly in the void volume of a size-exclusion chromatography column (Fig. 4b-c) and consistent with this, negative-stain images showed that NOD2 $\triangle$ LRR forms soluble aggregates (Fig. 4d). We mixed MBPRIP2CARD with a less aggregated fraction of NOD2 $\triangle L R R$ (Fig. 4b, d) and induced filament polymerization by addition of TEV. We then applied immuno-gold labelling against NOD2. As a control, we applied the same immuno-gold labelling to the NOD2 $\triangle$ LRR sample in the absence of RIP2CARD (Fig. 4d). The control demonstrates that with the protocol used, NOD2 $\triangle L R R$ can be specifically labelled, although with a heterogeneous number of gold-particles bound per aggregate. Micrographs of the NOD2 $\Delta$ LRR sample mixed with RIP2CARD showed 

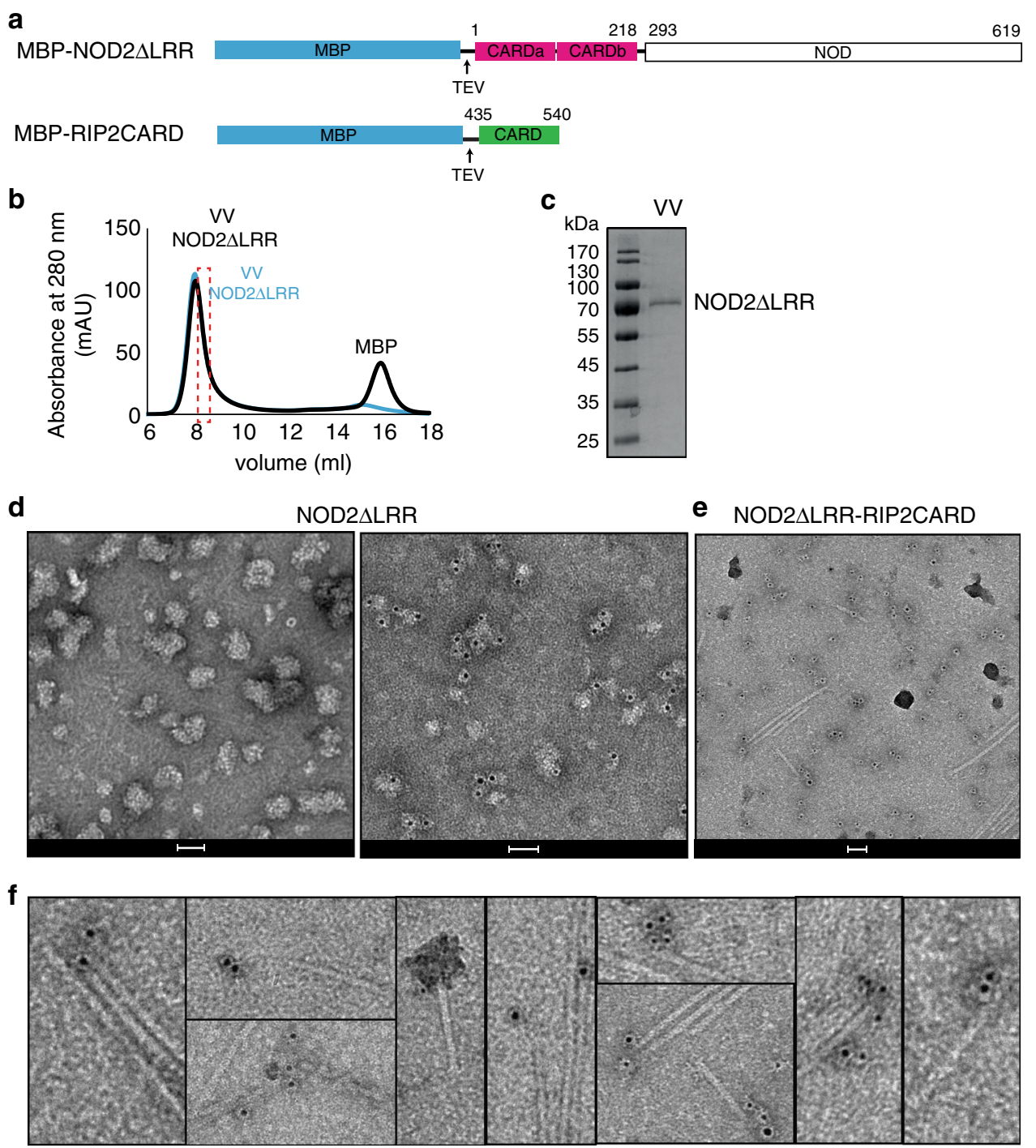

Fig. 4 NOD2 $\Delta$ LRR binds RIP2CARD filaments. a Domain organization of NOD2 $\Delta$ LRR and RIP2CARD constructs used for immuno-gold labelling experiments. Expression of recombinant NOD2 $\Delta$ LRR was done in sf21 insect cell. b, c Size exclusion chromatography profile (b) and (c) corresponding $12.5 \%$ SDS-PAGE showing typical sample obtained from NOD2 $\Delta$ LRR purification. The size exclusion chromatography profile shows that both tagged (blue) and tag-free NOD2 $\Delta$ LRR (black) elute in the VV. The dashed rectangular shape indicated the protein fraction used for immuno-gold labelling experiments. d Negative-stain images of VV NOD2 2 LRR, corresponding to the fraction used reconstitution with RIP2CARD, unlabelled (left) and after immuno-gold labelling (right). e, f Example negative-stain micrograph of RIP2CARD filaments with NOD2 $L$ LRR bound (e) and zoom showing gold-particles (black dots) after immuno-gold labelling against NOD2 (f). Scale bars are $50 \mathrm{~nm}$. VV: size exclusion chromatography void volume

gold-particles on individual NOD2 $\triangle \mathrm{LRR}$ aggregates or NOD2 $\Delta$ LRR aggregates bound to RIP2CARD filaments, mostly at one filament-end (Fig. 4e-f).

We then investigated by co-purification and immuno-gold labelling whether the NOD2CARDS are sufficient to interact with the RIP2CARD filament. For this, we used NOD2CARDS (residues 1-218) expressed with a cleavable N-terminal HISSUMO tag and a C-terminal SNAP tag (HIS-SUMO-NOD2$\mathrm{CARDS}^{\mathrm{S}}$ ) together with cleavable MBP-RIP2CARD (Fig. 5a). Due to the different requirement for optimal expression of these two constructs, we expressed them separately in E. coli and then mixed the pellets and co-purified the proteins. After clarification of the crude extract by centrifugation, the supernatant was applied to amylose resin and the eluate was analysed by SDSPAGE and western blot (WB), using a specific antibody against the SNAP tag (Fig. 5b-c). The results showed that HIS-SUMONOD2CARDS ${ }^{S}$ co-elute with MBP-RIP2CARD (Fig. 5b-c). The diameter of filaments observed by negative-stain EM after HISMBP tag cleavage was the same as the homo RIP2CARD filaments, while their length ranged from 50 to $500 \mathrm{~nm}$ (Fig. 5d).

We then applied immuno-gold labelling against the SNAP tag. The results showed single gold-particles mostly sitting on filament-ends (Fig. 5d-e, Supplementary Fig. 6). In order to evaluate the binding position of NOD2CARDS $S$ on RIP2CARD filament, two more controls were performed with the same immuno-gold labelling protocol: immuno-gold labelling on RIP2CARD filament without NOD2CARDS ${ }^{S}$ (Control 1, C1) and immuno-gold labelling with only the secondary antibody on NOD2CARDS ${ }^{S}$-RIP2CARD filament (Control 2, C2) (Supplementary Fig. 6c, d). Control 1 was used to evaluate the specificity of the primary antibody, whilst Control 2 was used to judge the specificity of secondary antibody. We collected 20 random images on two different grids for each condition at a magnification of $\times 16,000$ and evaluated the number and position of gold-particles 
a

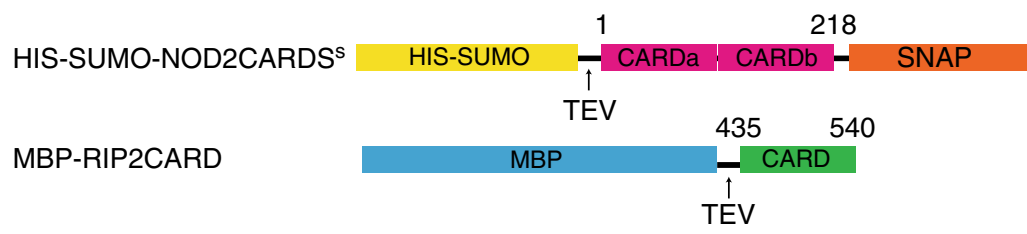

b
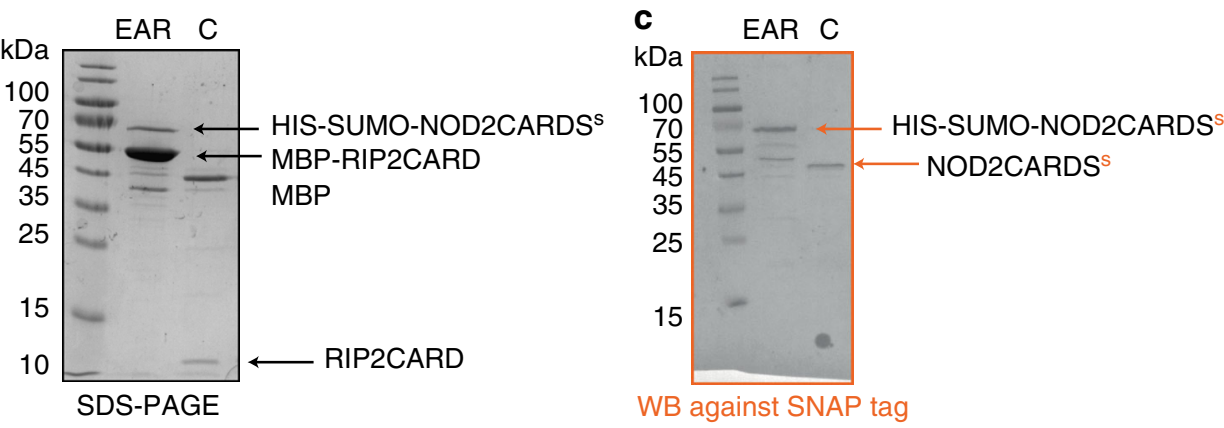

d
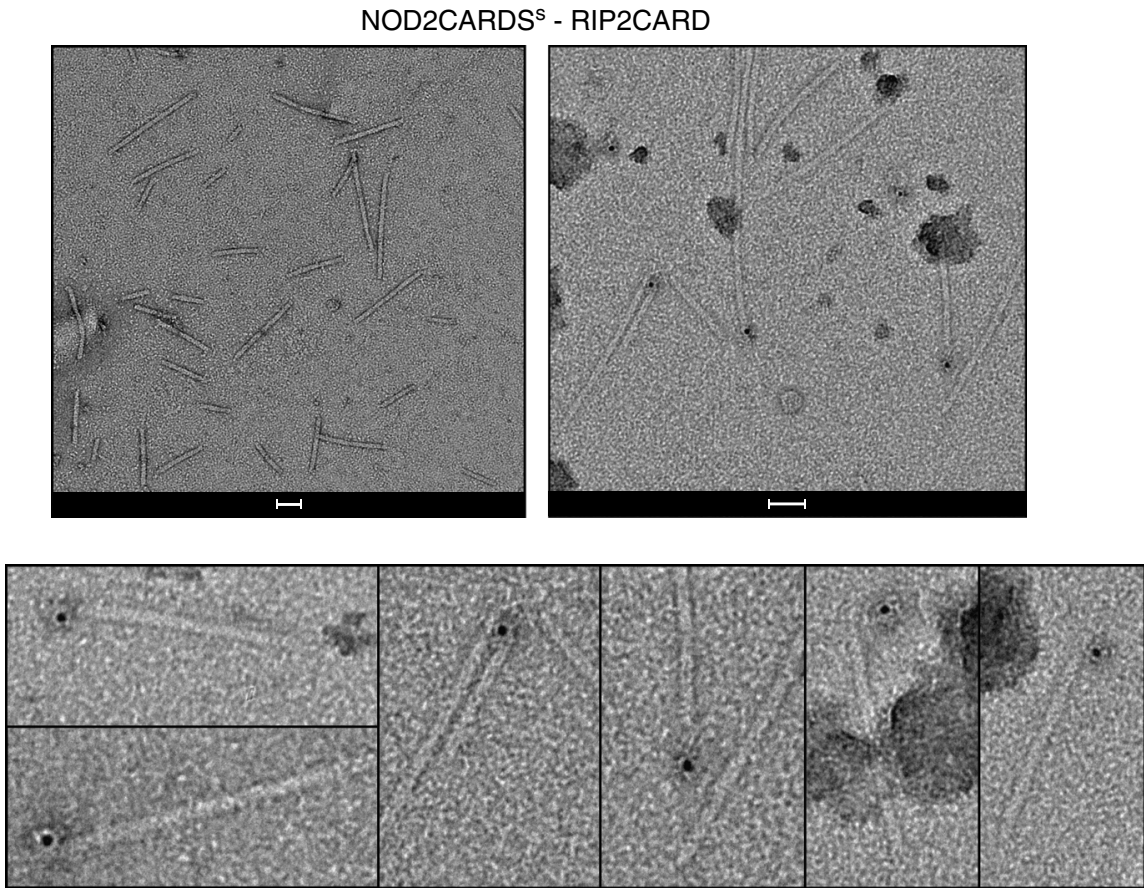

Fig. 5 NOD2CARDS bind at one end of the RIP2CARD filaments. a Domain organization of HIS-SUMO-NOD2CARDS and RIP2CARD constructs used for immuno-gold labelling experiments. Expression of recombinant of HIS-SUMO-NOD2CARDS was carried out in E. coli Rosetta 2. b, c 17\% SDS-PAGE (b)

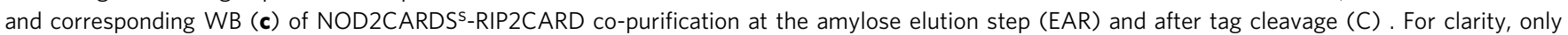
relevant lanes are labelled. d Negative-stain image of RIP2CARD filaments with NOD2CARDS bound, unlabelled (left) and after immuno-gold labelling (right). Scale bars are $50 \mathrm{~nm}$. e Zoom on micrographs of RIP2CARD filaments with NOD2CARDS s bound, showing gold particles (black dots) after immuno-gold labelling against the SNAP tag attached to NOD2CARDS

in each micrograph (Supplementary Fig. 6f). Final statistics revealed that $70.9 \%$ of gold-particles are found on filaments, of which $91.7 \%$ are at one end and we never observe gold-particles at both ends. This shows that NOD2CARDS ${ }^{5}$ are preferentially bound at one end of the RIP2CARD filament. These data are consistent with the hypothesis that under physiological conditions activated NOD2 nucleates RIP2 filament formation yielding a polar assembly.

Cryo-EM of RIP2CARD filament. To elucidate the architecture of the RIP2CARD filament by cryo-EM, we optimized the protocols for production of both the RIP2CARD and NOD2CARDS $^{S}$-RIP2CARD filaments (Supplementary Fig. 7). For this we used a different RIP2CARD construct encoding for RIP2CARD (residues 431 - 540) with a P3C (human rhinovirus 3C protease) cleavable HIS-MBP tag at the N-terminus (HIS-MBPRIP2CARD) (Supplementary Fig. 7a). This new RIP2CARD construct dramatically increased the tag cleavage efficiency (compare Fig. 3c with Supplementary Fig. 7c, f). We optimised the purification protocol for NOD2CARDS ${ }^{S}$-RIP2CARD filaments, by introducing a size exclusion chromatography step before tag cleavage (Supplementary Fig. 7b). This allows aggregates to be discarded and the tagged NOD2CARDS - RIP2CARD complex to be separated from monomeric HIS-MBP-RIP2CARD.

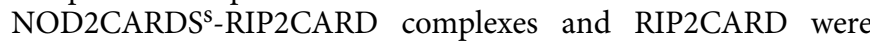
then recombined as described in the Methods. After cleavage, 
HIS-MBP, HIS-SUMO tags and HIS-tagged proteases, were removed by affinity chromatography followed by dialysis with a high molecular weight cut off (Supplementary Fig. 7c). RIP2CARD filaments were prepared following the same protocol (Supplementary Fig. 7f). Negative-stain and cryo-EM micrographs of samples prepared under the same conditions show that RIP2CARD filaments with bound NOD2CARDS ${ }^{s}$ are shorter, straighter and have a lower tendency to aggregate than RIP2CARD filaments without NOD2 (Supplementary Fig. 7d, e, g, h). Therefore, the hetero-CARD filaments were used for cryo-EM data collection and analysis (Supplementary Table 3).

Visual inspection of the individual cryo-EM images, 2D classaverages and corresponding power spectra indicate that the RIP2CARD filament has a helical symmetry (Fig. 6a-c). Indexing of the power spectra and symmetry refinement revealed a lefthanded helix of 3.56 subunits/turn with an axial rise of $4.848 \AA$ / subunit (Supplementary Fig. 8a, b). The final cryo-EM map at $3.94 \AA$ resolution (Supplementary Fig. 8c) shows that the filament has an approximate outer diameter of $\sim 75 \AA$ with a central solvent channel of $\sim 25 \AA$ diameter (Fig. 6d). The crystal structure of RIP2CARD can be unambiguously fitted into the cryo-EM density, with both $\mathrm{N}$ - and C-terminal ends orientated towards the outside of the filament (Fig. 6e). Manual adjustment using the clear density for the $\alpha$-helices and the larger side chains such as tryptophan and arginine (Fig. 6f), followed by real space refinement, led to a final atomic model which comprises RIP2CARD residues P433 to Q518 (Supplementary Table 3). This shows that, consistent with the solid-state NMR results, there are only minor structural re-arrangements of the CARD domain upon filament formation (RMSD of $1.12 \AA$ for $\mathrm{Ca}$ positions of residues 433-518) and the presumed flexible Cterminus is not observed in the EM map.

Structure of the RIP2CARD filament. The RIP2CARD filament has a similar helical configuration to other CARD filaments already described, such as MAVS CARD, Caspase-1 CARD and the recently described BCL10 CARD filament ${ }^{30,31,34,35}$ (Supplementary Table 4). Following the established convention, the RIP2CARD assembly can be described through interactions at three major asymmetric interfaces, named type I, type II and type $\mathrm{III}^{47}$. Type I and II are inter-strand interactions, whilst type III is intra-strand along the helical strand trajectory (Fig. 7a-c).

The type I interface is defined as the interaction between helices $\mathrm{H} 1$ and $\mathrm{H} 4$ of one molecule (type Ia surface) with $\mathrm{H} 2$ and $\mathrm{H} 3$ of the adjacent one (type Ib surface). In the RIP2CARD filament, the type I interaction is electrostatic in nature involving several charged residues that form polar interactions (Fig. 7d). D461 and N457 from H3 (type Ib) interact with R444 and R448 from $\mathrm{H} 1$ and $\mathrm{H} 4$ (type Ia) respectively. The interaction between $\mathrm{H} 1$ (type Ia) and H2 (type Ib) is further reinforced by backbone contacts between the break in $\mathrm{H} 1$ and the C-terminus of $\mathrm{H} 2$ (Fig. 7d, Supplementary Fig. 9a-b). Additional charged or polar residues such as D492, D495, Q441, E445 and Q489 (type Ia) and D467, K471 (type IIb) contribute to the type I interface (Fig. 7d).

The type II interface is normally defined by the interaction between the C-terminal end of $\mathrm{H} 4$ and the H4-H5 loop (type IIa surface) and a groove defined by the $\mathrm{H} 1$ and $\mathrm{H} 2$ corner from one side and H6 helix and its preceding loop on the other (type IIb surface). In the case of the RIP2CARD, which lacks H6, we identified a somewhat different type II interface. The type IIa surface includes the C-terminal end of $\mathrm{H} 4$, the $\mathrm{H} 4-\mathrm{H} 5$ and $\mathrm{H} 2$ $\mathrm{H} 3$ loops, whilst the type IIb surface comprises the H1-H2 loop, the N-terminal of $\mathrm{H} 2$ and the visible part of the RIP2CARD Cterminal (Fig. 7e). At the type II interface, the side-chains of M470 (type IIa) and C455 (type IIb) make van der Waals interactions (Fig. 7e and Supplementary Fig. 9c), these side-chains being specific to RIP2CARD (Supplementary Fig. 4). This interface is further reinforced by a polar interaction between N512 and Q458 (Fig. 7e). Moreover, Q497 (type IIa) can interact with the side chains of Q450, T452 and Q514 (type IIb) (Fig. 7e).

The type III interaction normally occurs between H3 (type IIIa) and a groove formed by $\mathrm{H} 1-\mathrm{H} 2$ and the H3-H4 loop (type IIIb). In the RIP2CARD filament, type IIIb comprises the H3-H4 loop and N-terminal of $\mathrm{H} 4$, whilst the H1-H2 loop is only contributing to the type IIa surface as described above (Fig. 7f). P481, T482 (type IIIa) and L476 (type IIIb) contribute hydrophobic interactions, whilst E472 and E475 from H3 (type IIIa) potentially form salt bridges with R488 and R483 (type IIIb). Interestingly, both side chain of T482, T484 could swap from the original position in the crystal structure to interact with type IIIa instead of contributing to the intra-ahelical interactions (Fig. $7 \mathrm{f}$ ).

Figure $7 \mathrm{~g}$ shows that the most significant chemical-shift differences between RIP2CARD in solution and within the filament, as determined by NMR, map to the subunit interfaces described above. These chemical-shift differences report on local conformational changes due to packing effects in the filament and therefore independently confirm the overall architecture of the intermolecular interfaces observed by cryo-EM. Notably, residues K443, R444, D495, L457, L456, K471 and Y474, located close at the type I interface, undergo strong chemical shift changes in the filament. Residue Y474 shows the largest effect. At its $\mathrm{C} \gamma$ resonance, multiple contacts in ${ }^{13} \mathrm{C}-{ }^{13} \mathrm{C}$ correlations employing long mixing times are observed, which indicates tight packing of Y474 in the filament. Similarly, conformational changes in the type IIa (M470) and type IIIb (R483, T484) residues lead to significant chemical shift changes of the respective residues, indicating a change in environment of these residues.

Mutational analysis of the RIP2CARD type II interface. Our immuno-gold labelling results show that NOD2CARDS ${ }^{S}$ bind at one end of the RIP2CARD filament, suggesting that an initial hetero-CARD complex might act as a nucleation point to promote unidirectional RIP2CARD filament growth. Available structures or models of hetero DD complexes, such as RIG-IMAVS, the Myddosome, the PIDDosome and NLRP3-ASC, show that the $\mathrm{DD}$ belonging to the receptor protein continues the helical arrangement of the effector DDs, by forming a combination of the same type I-II-III interfaces. With a view to testing the effect on signalling of site-directed mutants that would uniquely disrupt the RIP2CARD filament structure and not the RIP2NOD2 hetero-CARD interaction, we modelled the hypothetical hetero-CARD type I, II, III interfaces and evaluated their importance based on the available interaction and mutagenesis studies $^{12,14}$ (Supplementary Fig. 10). As the structure of NOD2CARDS is not yet available and the only reported direct interactions are to the N-terminal NOD2 CARDa (residues 26-122), we computed a three-dimensional NOD2CARDa model using Swiss Modeller ${ }^{48}$ using as template the X-ray structure of the CARD of Nucleolar Protein 3 (PDB code:4UZ0) ${ }^{49}$, which shares $35 \%$ identity with NOD2CARDa (Supplementary Fig. 10a), The NOD2CARDa model structure obtained is similar to RIP2CARD (RMSD of $1.70 \AA$ for all Ca positions), consistent with the presumed compatibility of both CARDS to form similar type I-II-III interactions.

NOD2CARDa residues R38, R86, E69, D70 and E72 and RIP2CARD residues E472, D473, E475, D461 and D492 residues have been shown to be important for hetero-CARD interactions $^{12,14}$. Our modelling shows that all these charged residues occur in type I or type III interfaces and are in fact highly conserved between NOD2CARDa and RIP2CARD 
a

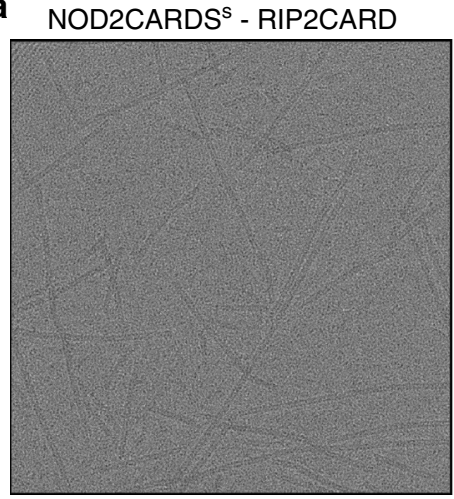

b

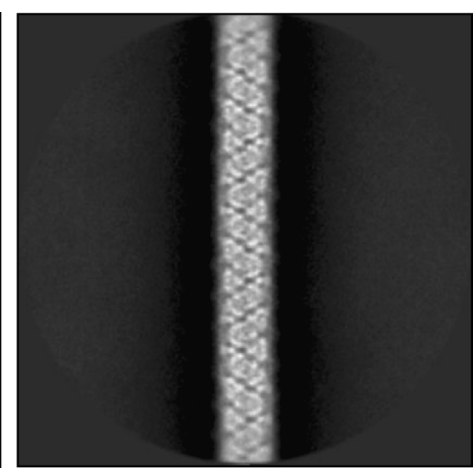

C

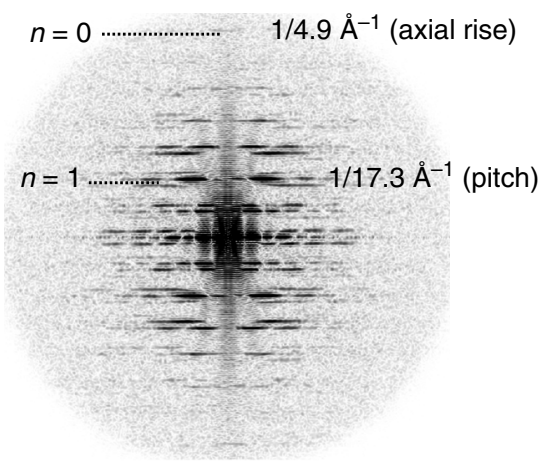

d

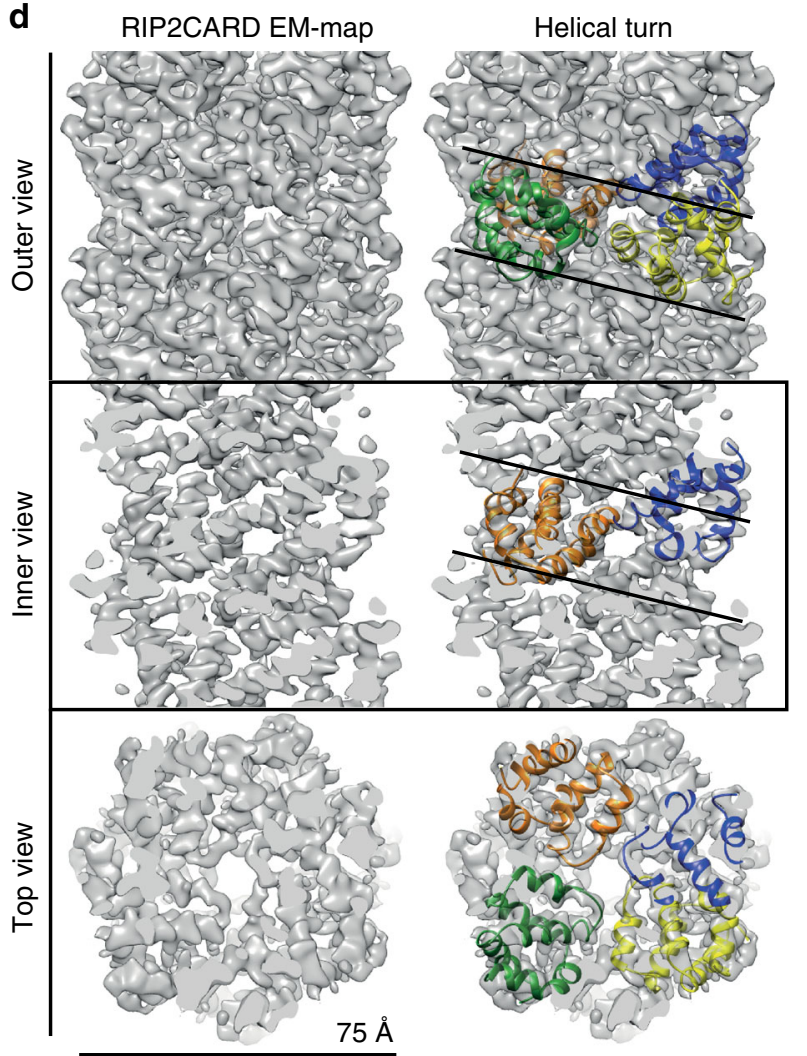

e

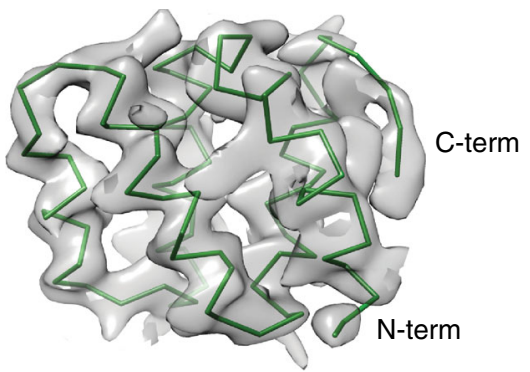

f

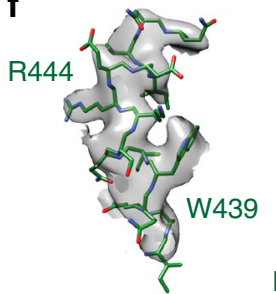

$\mathrm{H} 1_{435-450}$

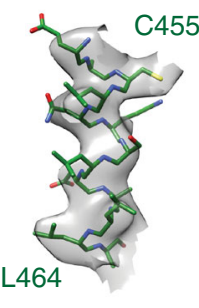

$\mathrm{H} 2_{453-465}$

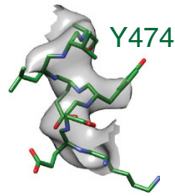

$\mathrm{H} 3_{471-478}$
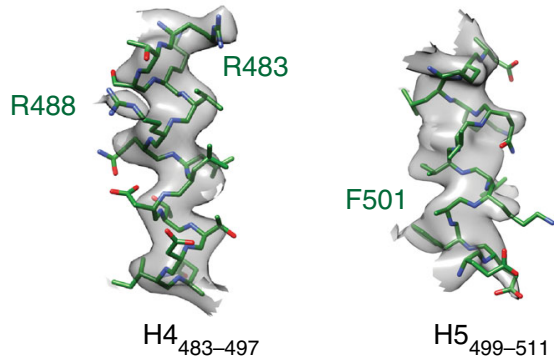

Fig. 6 Cryo-EM structure of the RIP2CARD filament. a Cryo-EM image of NOD2CARDSS-RIP2CARD filaments used for structure determination. $\mathbf{b}$, $\mathbf{c} 2$ Dclass average (b) and corresponding power spectra (c) used for initial symmetry parameter estimation. $\mathbf{d}$ Final cryo-EM map of the three-dimensional RIP2CARD filament at $3.94 \AA$ resolution (FSC in Supplementary Fig. 8c). Outer, inner and top view without (left) and with (right) RIP2CARD models fitted into one helical turn. $\mathbf{e}$ View of the RIP2CARD monomer fitted into the EM map showing only the main chain for clarity. $\mathbf{f}$ Zoomed-in views of the fitting of individual $\alpha$-helices into the sharpened cryo-EM density. Helices are defined as in the sequence alignment (Supplementary Fig. 4) except for $\mathrm{H} 1$, which starts from 1435 in the RIP2CARD monomer within the filament. Sidechains are shown as stick models. A few residues with clear EM density for sidechains are labelled

(Supplementary Fig. 10b-c, and Supplementary Fig. 4). Therefore, mutagenesis of RIP2 residues involved in these interfaces would affect both the interaction with NOD2 and filament formation. Conversely, none of the residues belonging to the observed RIP2 homo-type II interface have been explicitly implicated in the NOD2CARD-RIP2CARD interaction. We deduced that type II interactions within the RIP2CARD filament, notably involving RIP2CARD specific hydrophobic residues C455 and M470, could specifically stabilise homo-interactions within the filament.
We therefore mutated the residues belonging to RIP2CARD type IIa and IIb surfaces (type IIa: M470 and Q497; type IIb: Q450, T452, E453, C455, Q458 and N512) by alanine and lysine substitution (serine for C455). We then assayed the ability of each mutated RIP2CARD construct to bind NOD2CARDS ${ }^{S}$, using the co-purification protocol described above (Fig. 8a, b). Mutant RIP2CARD domains were expressed at similar level to wild-type RIP2CARD and all the mutants displayed unimpaired binding to NOD2CARDS , except for RIP2CARD T452K, which clearly 
a

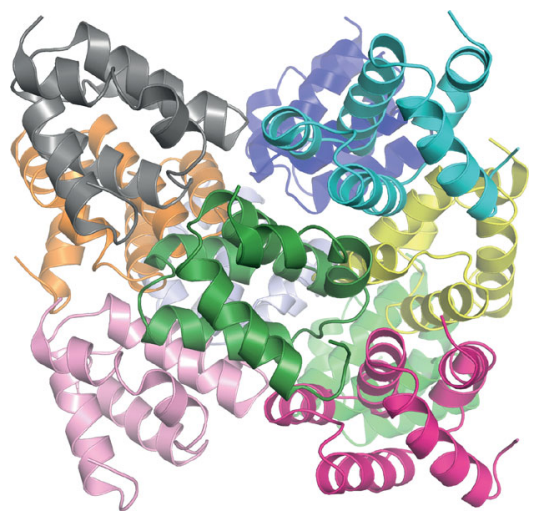

d

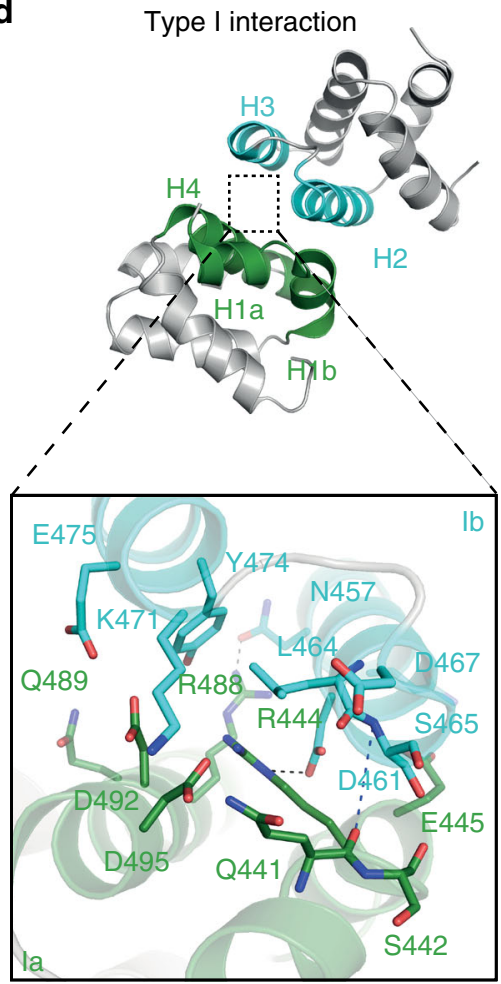

b

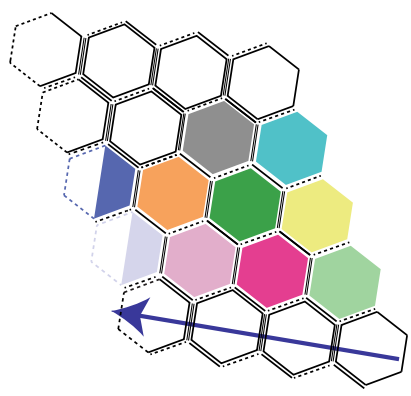

Helical strand direction c

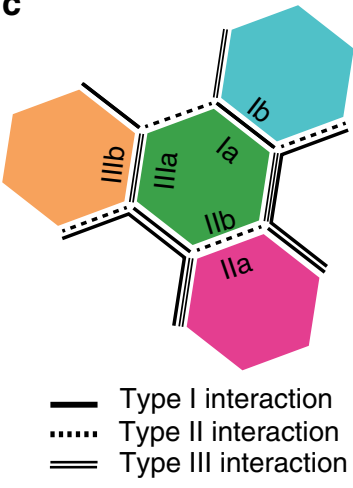

e Type II interaction

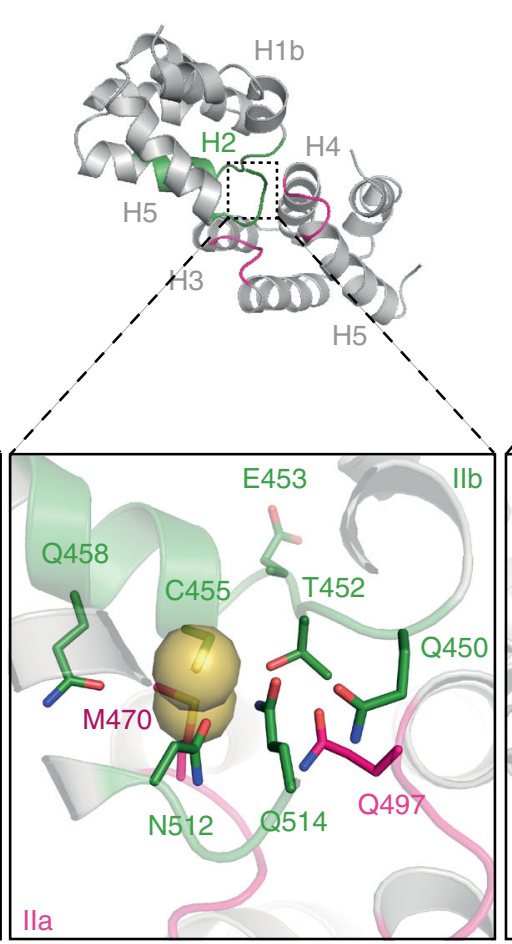

f

Type III interaction

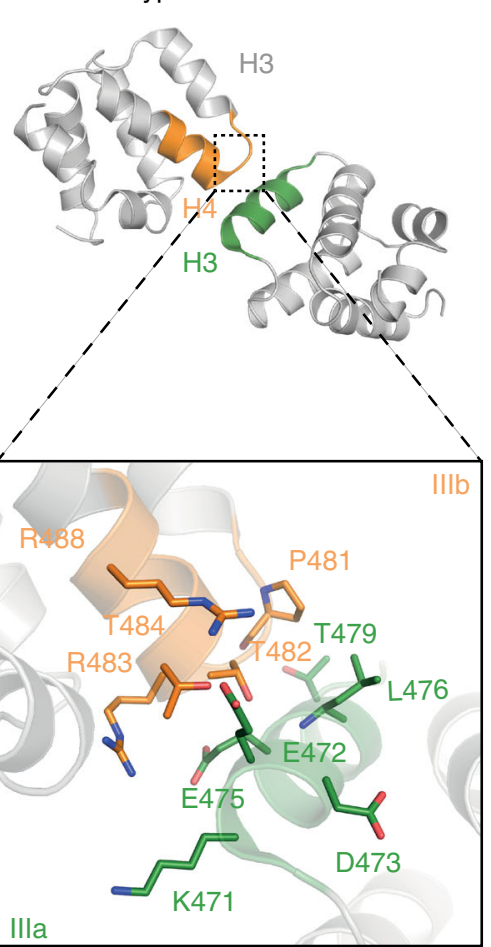

g

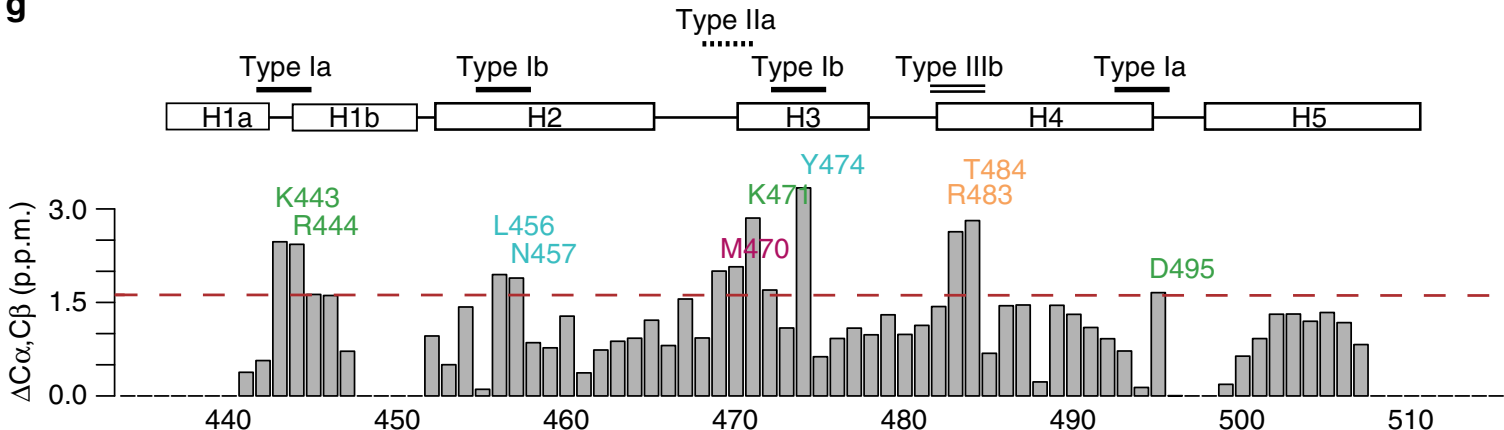

Fig. 7 Structural analysis of the RIP2CARD filament assembly. a Ribbon diagram of RIP2CARD filament comprising 10 subunits. b, c Schematic diagram of the helical filament (b) and relative orientations of type I, type II and type III interfaces (c). Each subunit is represented as a hexagon with the same colour code as in the filament structure in (a). Each turn comprises 3.56 subunits. The fourth subunit is represented as a half-empty hexagon to highlight that it is shared with the next turn. Type I, II, III interfaces are represented as a single line, single-dashed line or double line respectively. $\mathbf{d}$-f Ribbon diagram of RIP2CARD dimers interacting through (d) type I, (e) type II and (f) type III interfaces. Protein regions involved in the interface are highlighted using the colours as in (c). The insets show the interactions at relative type surface. $\mathrm{H}$-bonds are represented by black dashed line. Backbone contacts are highlighted by blue dash line, respectively. Sulphurous groups involved in Van der Waals interactions in the type II surface are represented as spheres. g Sum of ${ }^{13} \mathrm{C} \alpha$ and ${ }^{13} \mathrm{C} \beta$ chemical shift differences between RIP2CARD in the solution and the solid state for each assigned amino acid. Residues that show a strong variation from the mean (1.2 p.p.m.; dotted red line) are labelled in the same colour code as used in (d-f). The secondary structure elements and positions of the interface surfaces are also shown 


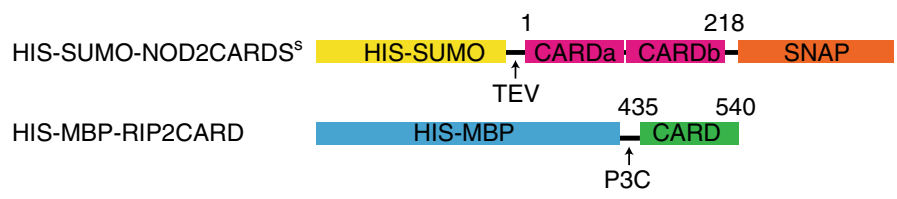

b

Type Ilb surface + HIS-SUMO-NOD2CARDS ${ }^{S}$
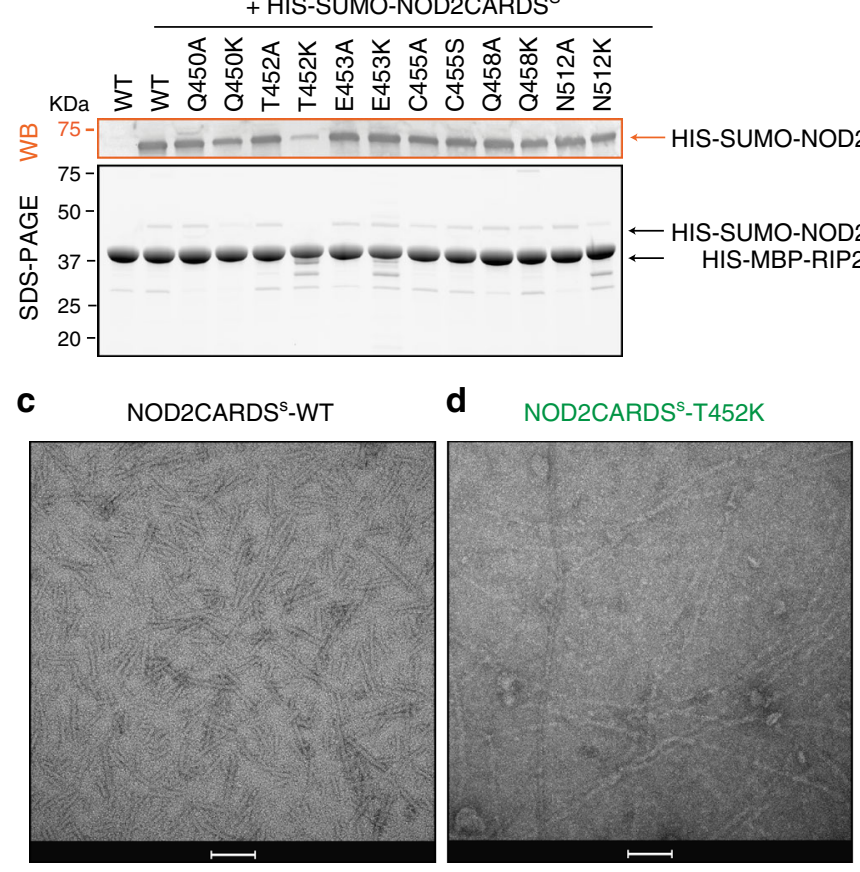

e NOD2CARDS ${ }^{\mathrm{S}}$-E453K

f

g

d

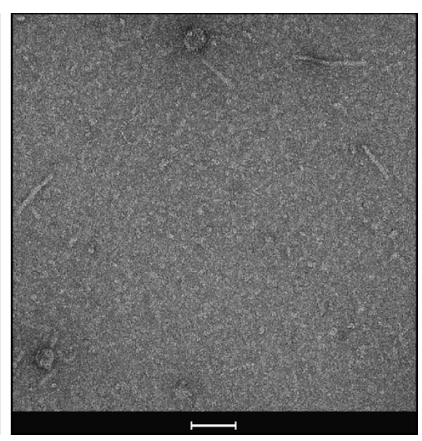

f NOD2CARDS ${ }^{\mathrm{S}}$-C455S

g NOD2CARDS $-M 470 A$

h NOD2CARDS ${ }^{\mathrm{S}}$-M470K
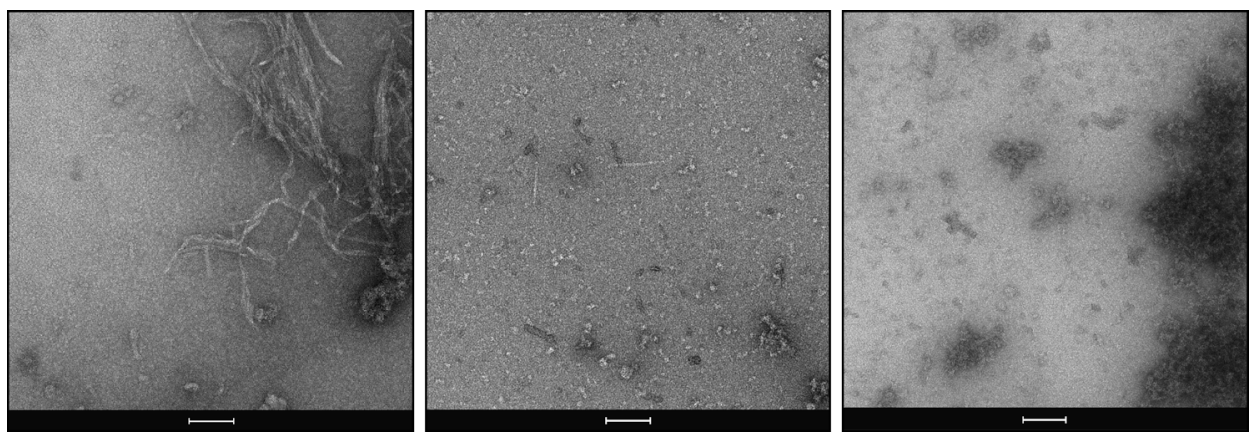

Fig. 8 Effect of type II surface mutations on RIP2CARD polymerization. a Domain organization of the NOD2CARDS and RIP2CARD constructs used for testing the behaviour of RIP2CARD mutants. b Western blot (WB, top panel) and 12.5\% SDS-PAGE (bottom panel) of NOD2CARDS ${ }^{5}$-RIP2CARD type II interface mutant co-purification at the amylose elution step (EAR). For clarity, only relevant lanes are labelled. Type Ila and Ilb surfaces are coloured as in Fig. 7. c-h Example micrographs of (c) NOD2CARDSs-WT, (d) NOD2CARDSs-T452K, (e) NOD2CARDSs-E453K, (f) NOD2CARDSs-C455S, (g) NOD2CARDS $-M 470 A$ and (h) NOD2CARDS'-M470K, after tag cleavage. Scale bars are $100 \mathrm{~nm}$

showed lower binding in comparison to wild-type and all the other mutants. We then tested the ability of each construct to polymerize after tag cleavage by imaging the sample with negative-stain EM. The micrographs revealed that many constructs can still polymerise (Supplementary Fig. 11), but with a dramatic change in the filament quality notably for mutants T452K, E453K, C455S, M470A and M470K (Fig. 8c-h). RIP2CARD-T452K forms long filaments but with multiple interruptions compared to wild-type and furthermore they have impaired binding to NOD2CARDS (Fig. 8b-d). This indicates that the mutation negatively affects both binding to NOD2CARDS $^{S}$ and filament quality. Micrographs of RIP2CARDE453K and RIP2CARD-M470A show particles that might correspond to protein aggregates with rare filaments (Fig. 8e, g). RIP2CARD-C455S forms irregular, more flexible filaments with a high tendency to aggregate (Fig. 8f). M470K micrographs show protein aggregation and absence of filaments (Fig. 8h).

We next investigated the effect of these mutations on the activation by NOD2 of transcription factor NF- $\mathrm{kB}$, by using a luciferase reporter assay (Fig. 9 and Supplementary Fig. 12). We transiently transfected HEK293T cells with HA tagged RIP2fl mutants (Fig. 9a) together with a plasmid encoding firefly luciferase under the control of NF-kB promoter ${ }^{50}$. NF- $\mathrm{kB}$ activation was induced using the specific NOD2 activator MDP, and cells were lysed $20 \mathrm{~h}$ later to record luciferase activity and assay protein expression. In agreement with the in vitro data, mutants Q450, Q458, Q497, N512 show unimpaired NF- $\mathrm{B}$ signalling; with the particularity that Q450K, Q497A and Q497K 

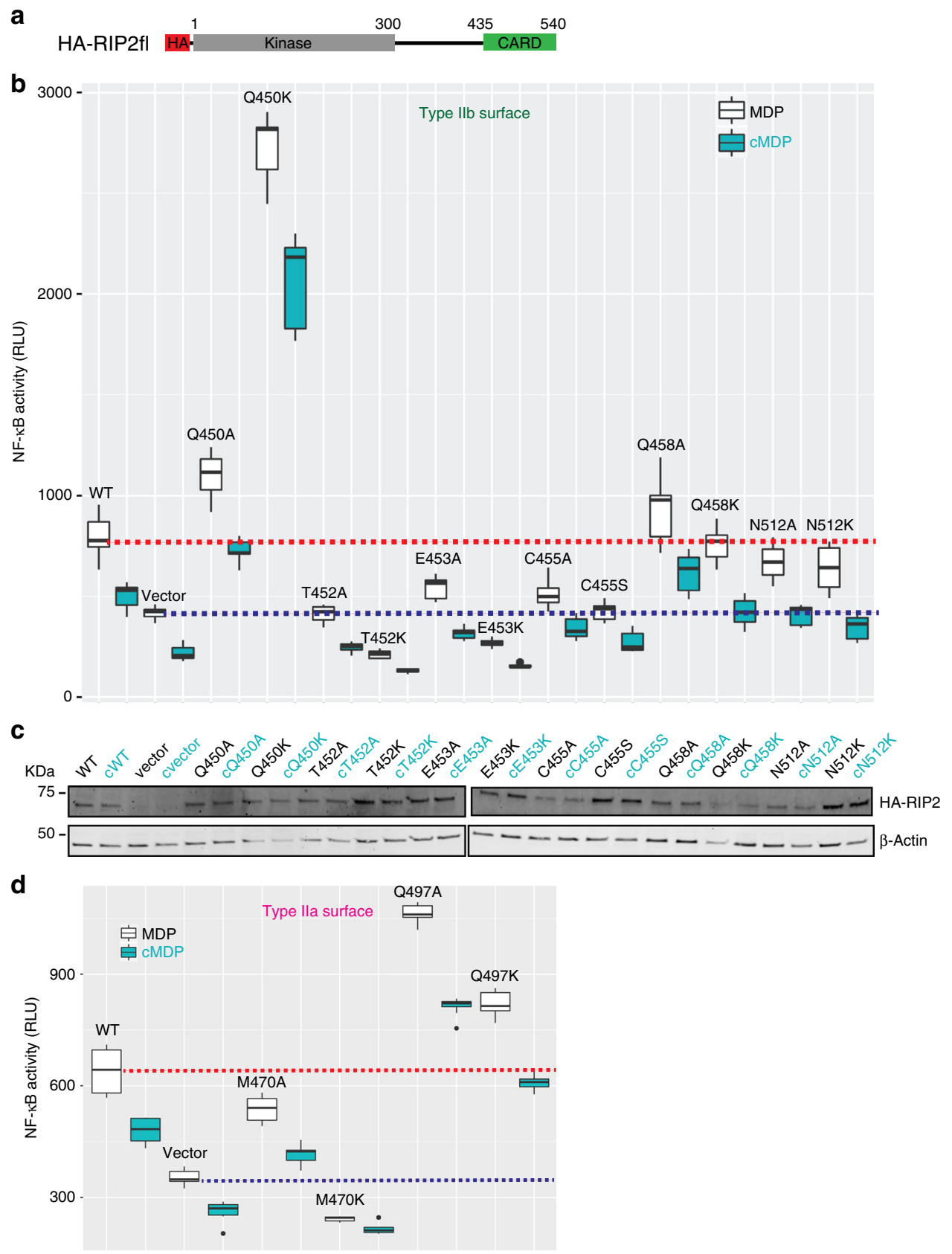

e

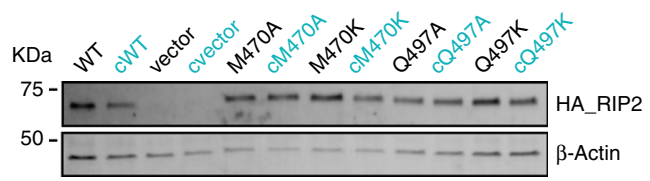

Fig. 9 Effect of type II surface mutations on NOD2 dependent NF-kB signalling. a Domain organization of the HA-RIP2fl construct used for the luciferase reporter assay. b, d Luciferase reporter assay in HEK293T cells showing the effect on NF-kB signalling of transfected WT and mutant RIP2fl for (b) type Ilb surface and (d) type lla surface. Each MDP value is significantly higher than that using c MDP. Average RLU values for WT RIP2fl-MDP and control-MDP are shown by dashed red and blue lines, respectively. The boxplot is the graphical representation of the summary statistics of a vector contains information in this order: minimum value (lower whisker), first quartile ( $25 \%$ of data lower bound box), median ( $50 \%$ of data centre line), third quartile ( $75 \%$ of data higher bound box) and maximum value (upper whisker). Black dots on boxplot are extreme values. c, e Example WB for (c) type Ilb surface mutants and (e) type lla surface mutants, showing expression of wild-type (WT) HA-RIP2fl and mutants (top) and $\beta$-actin (bottom). For each construct, the results for cells induced by either MDP or its control (cMDP, c) are reported in black and cyan respectively

show high levels of auto-activation compared with the wild-type RIP2 (Fig. 9b, d). In contrast, T452K, E453K, C455S and M470K failed to transmit the signal from NOD2 to NF- $\kappa \mathrm{B}$, as the luciferase value is equal or lower than the control empty vector
(Fig. 9b, d). The less drastic alanine mutants T452A, E453A, C455A and M470A also showed lower or zero activity (Fig. 9b, d). All the mutants show similar protein expression to wt RIP2 (Fig. 9c, e and Supplementary Figure 12). 


\section{Discussion}

Recent studies on several innate immune systems have shown that recognition of cognate ligands by PRRs that contain a DD (e.g. CARD and PYD) triggers their oligomerization and interaction with the downstream adaptor resulting in the formation of a higher-order filamentous assembly called a signalosome $e^{29,51}$. Here we focus on the NOD2-RIP2 signalling pathway, a receptoradaptor protein combination that shares close structural similarities with PRRs involved in signalosome formation. Specifically, we investigated whether the recruitment of RIP2 by NOD2 via CARD-CARD interactions could lead to the formation of such a signalosome ('nodosome'). Our biophysical, structural and functional data show that RIP2, via its CARD, can form helical filaments, plausibly nucleated from one end by activated NOD2. Furthermore, we show that RIP2 polymerization is essential for NF- $\mathrm{kB}$ activation by NOD2, presumably by favouring recruitment of downstream effectors such as the RIP2 ubiquitin ligase $\mathrm{XIAP}^{17}$

The starting point was our finding that phosphorylated and active RIP2fl forms filaments in vitro in the presence of ATP and magnesium. The subsequent observation that RIP2CARD also spontaneously forms more slender filaments, suggests that the CARD domain forms the core of the RIP2fl filaments, while the kinase domain (RIP2K) is on the exterior. Interestingly we observed that not only ATP, but also non-hydrolysable adenosine nucleotides together with magnesium promote polymerization of RIP2fl. This suggests that enhanced RIP2 polymerization by nucleotide-binding results from RIP2K structure stabilization rather than any increase in RIP2K autophosphorylation activity. Our previously published biophysical data $^{36}$, show that stable activation of RIP2K involves the coupling of kinase dimerization with auto-phosphorylation of the activation loop. We therefore speculate that CARD polymerization promotes kinase dimerization, by increasing the local RIP2 concentration. Dimerization favours the kinase domain being in the active conformation and therefore able to bind any adenosinederived nucleotide, independently from the phosphorylation state of the activation loop. In a physiological context, RIP2 could either be already phosphorylated, as it was reported that autophosphorylation contributes to protein stabilization ${ }^{36,52}$ or further phosphorylated upon dimerization to stabilize the active conformation. These observations are in line with a recent study showing that an active conformation of the RIP2 kinase, rather than necessarily a catalytically active kinase, is essential for NOD2 signalling, since it permits interaction with the E3 ligase $\mathrm{XIAP}^{17}$.

In order to investigate the assembly mechanism of the RIP2 filament core, we co-purified RIP2CARD and NOD2CARDS and used immuno-gold labelling to show that NOD2CARDS bind preferentially at one of the two filament-ends, forming a polar assembly. We then successfully imaged this sample using cryoEM, obtaining an EM density map at $3.94 \AA$ resolution, where we could unambiguously fit and refine the X-ray structure of RIP2CARD, also reported in this paper. Solid-state NMR confirmed the absence of an ordered structure for the C-terminal segment in the filaments, involving residues beyond K510. RIP2CARD assembles into a left-handed helical filament with 3.56 subunits per turn, a configuration similar to that described for filaments of MAVS, Caspase-1 and, most recently, BCL10 CARDs ${ }^{30,31,34,35}$. RIP2 thus assembles differently from RIP1 and RIP3, which polymerize through their RHIM (RIP homotopic interaction motif) domain into amyloid fibrils ${ }^{53}$.

RIP2CARD assembles into a helical arrangement using the conventional type I, II and III interfaces. Consistent with this, Ca$\mathrm{C} \beta$ chemical shift differences between the solution and solid-state are the most significant for the residues involved at these interfaces (Fig. 3g). The cryo-EM structure reveals that the type I and type III interfaces are polar (Fig. 7d, f), mediated by interactions of R488 and R444 (type Ia surface) with N457 and D461 (type Ib), and by contacts between E472 and E475 (type IIIa surface) and R483, R488 and T484 (type IIIb). The type II interaction is more hydrophobic for instance involving C455 and M470 (Fig. 7e). Y474, which has the highest chemical shift change, is observed buried in the type I interface (Fig. $7 d$ ), suggesting that it is not accessible for phosphorylation in the filament. Nonetheless, it is reported that auto-phosphorylation of this residue is important for NOD2 signalling 54 , but might occur at a later stage, after RIP2 ubiquitination by $\mathrm{cIAP} 1^{54}$.

We then tested the relevance of RIP2 polymerization on the activation of NF- $\kappa$ B by NOD2, by designing RIP2CARD mutants that potentially affect RIP2 polymerization but preserve the interaction with NOD2. Based on available structures of heteroCARD complexes ${ }^{31,47}$, we expect that a cluster of NOD2CARDS from activated and oligomerized NOD2 could form a short helical extension that preserves the same helical parameters as the RIP2CARD filament. By combining this premise with available mutagenesis data, we deduced that the observed type II interface in the filament, unlike the type I or III interfaces, may be specific to the RIP2 homo-CARD interaction (Supplementary Fig. 10). Therefore, we performed mutagenesis of the RIP2 type II surface with the aim of finding mutants that specifically disrupt homo-, but not hetero-CARD interactions, thus allowing assessing the biological significance of the extended filament. Results from our structure-guided mutagenesis, show that RIP2CARD mutations to lysine (or serine for C455) at the type II interface can affect either hetero- and homo-CARD interactions or only homoCARD domain interactions. For instance, the T452K mutation both decreased the interaction with NOD2 (Fig. 8b), led to aberrant filament formation (Fig. 8d) and severely affecting signalling (Fig. 9b). On the other hand, mutations of E453, C455 and M470 did not disrupt the interaction with NOD2 but severely affected the quality of RIP2 polymerization and abolished signalling (Fig. 8b, e-h and Fig. 9b, d). The E453 side chain points towards the central channel, suggesting that the lysine mutation disfavours filament elongation by affecting the inner filament electrostatics. Similar reverse charge mutations at the type II interfaces in MAVS CARD filament, combined with others, abrogate MAVS filament elongation ${ }^{31}$. The C455S and M470A/K mutations would reduce the hydrophobic interaction at the RIP2CARD type II interface, consistent with their strong effect on the quality of filaments or whether filaments form at all (Fig. 8f-h). Interestingly, some alanine mutants (e.g. T452A, E453A and C455A) also show a significant decrease in the NF- $\kappa \mathrm{B}$ signal, despite the fact that they do not affect the ability of RIP2CARD either to bind NOD2CARDS or to polymerize in vitro. We speculate that alanine mutations have a milder effect, compared to changing the charge, that cannot be detected by negative-stain EM, except for the case of M470A, whose severe disruptive affect highlights the importance of this RIP2 specific residue in forming the type II interface in the filament.

In conclusion, we provide evidence for the existence and biological importance of a NOD2-RIP2 polar filamentous assembly, which is likely the core of the nodosome complex. Based on the results described here, other published data and analogy to other signalosome systems, we propose that nodosome assembly occurs as follows (Fig. 10): (1) binding of MDP to NOD2 LRR domain activates the receptor causing derepression of the CARDs; (2) NOD2 oligomerises via its NOD and CARD domains; (3) oligomerized NOD2 recruits RIP2 via its CARD domain forming the hetero-CARD complex (4) cumulative binding of RIP2 to the hetero-CARD complex promotes filament elongation to form the helical assembly here described; (5) polymerization of RIP2CARD 


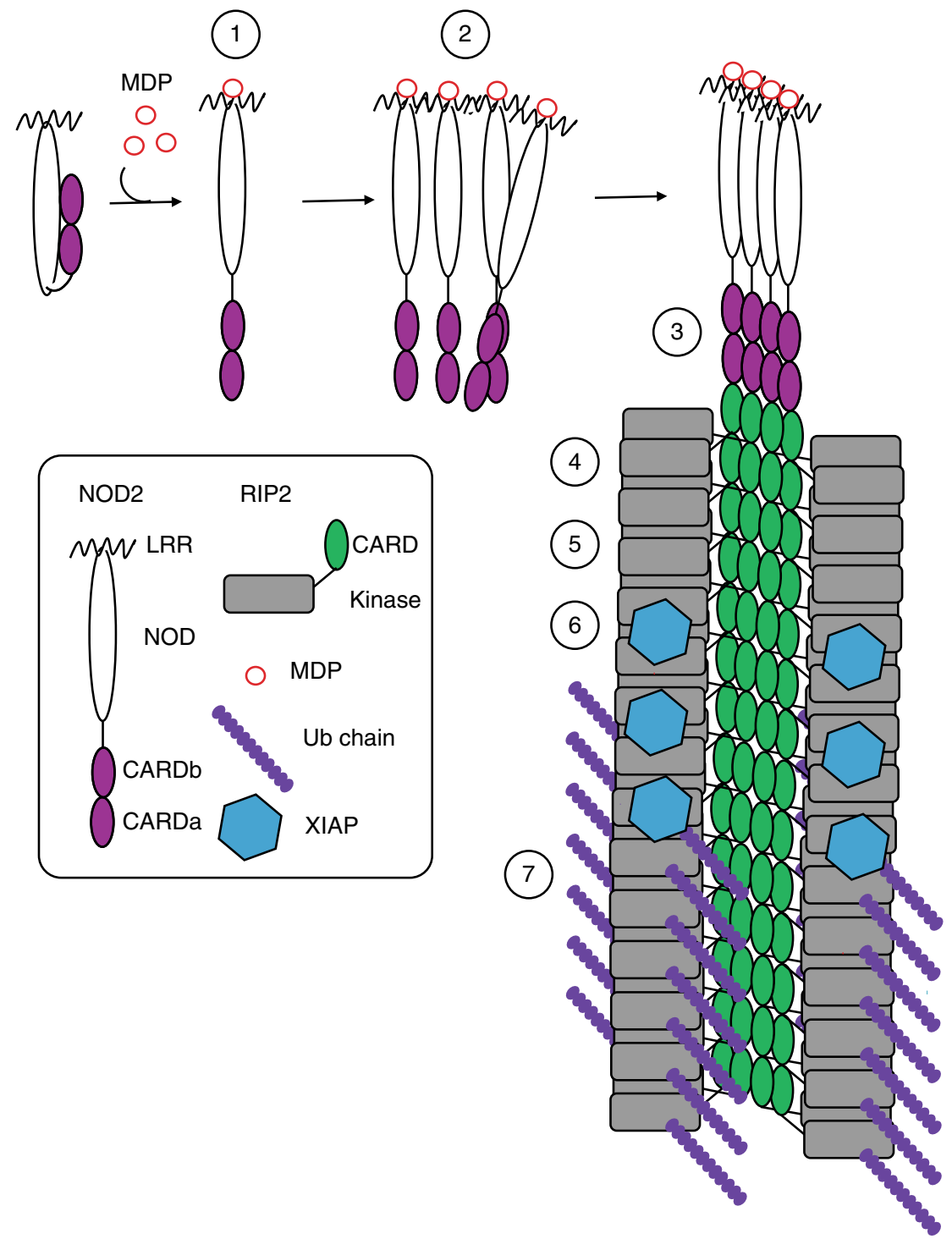

Fig. 10 Model of nodosome assembly. Model of nodosome assembly, based on the results described here, other published data and analogy to other signalosome systems. Steps 1-7 in the process are discussed in the main text. The legend for the components of the system is in the inset

in the presence of ATP stabilises the active antiparallel dimeric form of RIP2K; (6) E3 ligases, such as XIAP bind the active from of RIP2 $\mathrm{K}^{17}$; (7) RIP2 becomes K63-ubiquitinated enabling it to recruit downstream effector proteins.

Finally, by combining our biophysical, structural and functional analysis with existing data, we provide essential information that could potentially be used to explore new therapeutic options for inflammatory diseases characterised by aberrant NOD2-RIP2 signalling.

\section{Methods}

Constructs, protein expression and purification. Constructs described in this paper were generated from pcDNA3 plasmids encoding human RIP2 and human NOD $2^{50}$. The N-terminally HIS-tagged TEV (Tobacco Etch Virus) and P3C (human rhinovirus 3C) proteases used in this paper were produced at the Protein Expression and Purification Core Facility at EMBL, Heidelberg, Germany.

Recombinant human RIP2fl and human NOD2 2 LRR(1-619) were produced using the baculovirus system in $s f 21$ insect cells. The DNA sequence of RIP2fl and NOD2 $\triangle$ LRR were cloned from pcDNA3 into the vector pFastBacHTB using NcoI and HindIII cloning sites. Using the In-Fusion cloning technology (Takara Clontech), the original TEV cleavable HIS tag was replaced with a TEV cleavable maltose-binding protein (MBP) tag, which improved both expression and stability of recombinant proteins during insect cells expression (MBP-RIP2fl and MBPNOD2 $\triangle$ LRR). MBP-RIP2fl and MBP-NOD2 $\triangle$ LRR were expressed and purified following the same protocol ${ }^{36}$.Virus generation and amplification, insect cell infection and protein expression were performed at the EMBL Eukaryotic Expression Facility. Briefly, Sf21 cells at $0.6 \times 10^{6}$ cells $\mathrm{ml}^{-1}$ were infected with a virus shot able to stop cells growing in $24 \mathrm{~h}$. On the fourth day post-infection, cells were harvested and re-suspended in $1 \times 10^{-1}(\mathrm{v} / \mathrm{v})$ ratio of lysis buffer $20 \mathrm{mM}$ Tris $\mathrm{pH} 7.5,50 \mathrm{mM} \mathrm{NaCl}, 2 \mathrm{mM} \beta$-mercapto-ethanol ( $\beta \mathrm{Me}), 0.01 \% \mathrm{NP} 40$ supplied with protease cocktail inhibitor (Complete, Roche). Using a douncer, cells were homogenized and afterwards centrifuged at $18,000 \times g$ for $30 \mathrm{~min}$. The resulting supernatant solution was incubated for at least $2 \mathrm{~h}$ with amylose-affinity chromatography resin (New England Biolabs), whilst gently shaking at $4{ }^{\circ} \mathrm{C}$. The fusion protein was then eluted using the same lysis buffer supplemented with $40 \mathrm{mM}$ maltose. Upon overnight TEV cleavage, either RIP2fl or NOD2 $\triangle$ LRR were applied to size exclusion chromatography, using a similar buffer composition of lysis buffer (20 mM Tris pH 7.5, $50 \mathrm{mM} \mathrm{NaCl}, 0.5 \mathrm{mM}$ TCEP).

For crystallization purposes, RIP2CARD (435-540), was cloned into pETXM1 plasmid using the NcoI and XhoI cloning sites, resulting in a protein construct with an N-terminal crystallisable MBP (crystMBP-RIP2CARD) spaced by a three alanine linker. The construct was expressed in E. coli Rosetta 2 (Novagen) by growing the bacterial culture at $37^{\circ} \mathrm{C}$ until an $\mathrm{OD}_{600 \mathrm{~nm}}$ of 0.6 and inducing with $0.3 \mathrm{mM}$ IPTG (isopropyl- $\beta$-D-1-thiogalactopyranoside) overnight at $16^{\circ} \mathrm{C}$. The cells where harvested, re-suspended in $11^{-1}(\mathrm{v} / \mathrm{v})$ ratio of lysis buffer $20 \mathrm{mM}$ Tris $\mathrm{pH} 8,50 \mathrm{mM} \mathrm{NaCl}, 2 \mathrm{mM}(\beta-\mathrm{Me})$ containing protease cocktail inhibitor (Complete, Roche) and lysed by sonication. The crude extract was centrifuged for $30 \mathrm{~min}$ at $18,000 \times g$ and the soluble fraction was applied to amylose-affinity chromatography resin (New England Biolabs) and purified as described above. After elution, the fusion protein was applied to a prepacked anion exchange chromatography column (GE Healthcare) with a 0 to $1 \mathrm{M} \mathrm{NaCl}$ gradient. The protein was further purified on 
a Superdex 200 size exclusion chromatography column (GE Healthcare), in buffer containing $20 \mathrm{mM}$ Tris $\mathrm{pH} 8$ and $50 \mathrm{mM} \mathrm{NaCl}$. The protein corresponding to the monomeric peak was used immediately for crystallization purposes.

For immuno-gold labelling experiments and solid state NMR, RIP2CARD (435-540) was cloned in pETM40, which results in RIP2CARD with N-terminal MBP tag (MBP-RIP2CARD). Protein was expressed in E. coli Rosetta 2 by growing the bacterial culture at $37^{\circ} \mathrm{C}$ until $\mathrm{OD}_{600 \mathrm{~nm}}$ of 0.6 and inducing with $0.3 \mathrm{mM}$ IPTG for $4 \mathrm{~h}$ at $18^{\circ} \mathrm{C}$. Protein was either purified as described for crystMBP-RIP2CARD, or combined with NOD2CARDS ${ }^{s}$ as described in the immuno-gold labelling section.

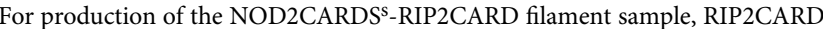
(431-540) was cloned into pETM41 using the in-Fusion cloning technology, which resulted in a fusion protein with N-terminal HIS-MBP tag (HIS-MBP-RIP2CARD). To improve the cleavage efficiency, the original TEV site was replaced by a P3C cleavage site. Protein was expressed as described for MBP-RIP2CARD except that media was supplied with $0.04 \%(\mathrm{w} / \mathrm{v})$ glucose to reduce bacterial MBP expression. The cells were harvested and either used for RIP2CARD filament production or combined with bacterial pellets containing HIS-SUMO- NOD2CARDSs protein to prepare NOD2CARDS ${ }^{\mathrm{s}}$-RIP2CARD filament sample.

NOD2CARDS (1-218) was cloned in pETM15 using the restrictions sites NcoI and XhoI. By applying the technology in-Fusion, a TEV cleavable HIS-SUMO tag was added to the N-terminus, while a non-cleavable SNAP tag was added to the Cterminus (HIS-SUMO-NOD2CARDS ${ }^{\mathrm{s}}$ ). The construct was transformed and expressed in E.coli Rosetta 2 by growing the bacterial culture supplied with $0.04 \%$ (w/v) glucose at $37^{\circ} \mathrm{C}$ until an $\mathrm{OD}_{600 \mathrm{~nm}}$ of 0.6 and inducing with $0.3 \mathrm{mM}$ IPTG overnight at $16^{\circ} \mathrm{C}$

For production of RIP2CARD filaments bound to NOD2CARDS ${ }^{s}, 1 \mathrm{~L}$ of RIP2CARD culture (or $\sim 5 \mathrm{~g}$ of bacterial pellet) was combined with $100 \mathrm{ml}$ of NOD2CARDS ${ }^{\mathrm{s}}$ (or $\sim 0.9 \mathrm{~g}$ of bacterial pellet) and resuspended in $100 \mathrm{ml}$ of lysis buffer $(20 \mathrm{mM}$ Tris $\mathrm{pH} 8,50 \mathrm{mM} \mathrm{NaCl}, 1 \mathrm{mM}$ TCEP and Complete proteases inhibitor cocktail (Roche)). The sample was then lysed by sonication and crude extract was let on gentle shacking for $30 \mathrm{~min}$ at $4{ }^{\circ} \mathrm{C}$. After centrifugation at $18,000 \times \mathrm{g}$ for $30 \mathrm{~min}$, the soluble fraction was incubated for $2 \mathrm{~h}$ with amyloseaffinity chromatography resin. The sample was then eluted using lysis buffer supplemented with $40 \mathrm{mM}$ maltose and complex formation was checked on SDSPAGE. The amylose eluate was successively passed through size exclusion chromatography column Superose6 (GE Healthcare) equilibrated in $20 \mathrm{mM}$ Tris $\mathrm{pH} 8,50 \mathrm{mM} \mathrm{NaCl}, 1 \mathrm{mM}$ TCEP to separate aggregates, NOD2CARDS $s_{-}$ RIP2CARD complex and RIP2CARD monomer. NOD2CARDS - -RIP2CARD complex at $0.25-0.35 \mathrm{mg} \mathrm{ml}^{-1}$ and monomeric RIP2CARD at $0.45-0.55 \mathrm{mg} \mathrm{ml}^{-1}$ were then combined at the desired ratio (usually $1 \times 10^{-1} \mathrm{v} / \mathrm{v}$ ) and filament formation was induced by adding protease overnight at $4^{\circ} \mathrm{C}$. HIS-MBP and HISSUMO tags, proteases and uncleaved protein were removed by applying the sample to a Ni-NTA resine (Takara). Further purification of filaments was achieved by dialysis overnight using a membrane with a $300 \mathrm{kDa}$ cut off. SDS-PAGE and negative-stain EM were used along the purification procedure to check sample homogeneity and filaments formation. Filaments containing only RIP2CARD were obtained and purified following a similar protocol. To avoid CARD protein aggregation all the purification steps were carried out at $4{ }^{\circ} \mathrm{C}$.

Gel filtration calibration curve. The calibration curve of the Superdex 200 column (GE Healthcare) used to purify RIP2fl, RIP2CARD and NOD2 $\triangle$ LRR (Supplementary Fig. 2) was produced by applying the instructions kit of Gel Filtrations Markers Kit for Protein Molecular Weights 29,000-7000,000 Da, (Sigma). To determine the Void Volume elution (VV), blue dextran was dissolved at concentration of $2 \mathrm{mg} \mathrm{ml}^{-1}$ in $20 \mathrm{mM}$ Tris $\mathrm{pH} 8,50 \mathrm{mM} \mathrm{NaCl}, 1 \mathrm{mM}$ TCEP and $5 \%$ Glycerol. Proteins Standards were mixed at ratio indicated in the instructions, in the same blue dextran buffer. $300 \mathrm{ul}$ of each sample were applied to the column, at $0.5 \mathrm{ml} \mathrm{min}{ }^{-1}$. The gel filtration runs were performed at room temperature in buffer containing $20 \mathrm{mM}$ Tris $\mathrm{pH} 8,50 \mathrm{mM} \mathrm{NaCl}$ and $1 \mathrm{mM}$ TCEP.

In vitro radioactive phosphorylation assay. We used an in vitro radioactive assay to analyse the auto-phosphorylation activity of recombinant RIP $2 \mathrm{fl}^{36} .1 .2 \mu \mathrm{g}$ of freshly purified RIP2fl were mixed with $10 \mu \mathrm{M}$ ATP $\left(10: 1\right.$ ATP-gamma- $\left.{ }^{32} \mathrm{P}\right)$ and $10 \mathrm{mM} \mathrm{MgCl}_{2}$. The reaction was incubated at $30^{\circ} \mathrm{C}$ and blocked at $1,3,5,10,15$ min by adding SDS loading buffer. Resulting samples were applied to $12 \%$ SDSPAGE gel and results were revealed using a Typhoon scanner (GE Health).

\section{Liquid chromatography/electrospray ionization mass spectrometry. The} phosphorylation profile of freshly purified and non-aggregated RIP2fl by Liquid Chromatography/Electrospray Ionization Mass Spectrometry (LC/ESI-MS), was analysed on a 6210 TOF mass spectrometer coupled to a HPLC system (1100 series, Agilent Technologies) ${ }^{36}$. The mass spectrometer was calibrated with tuning mix (ESI-L, Agilent Technologies) and the following settings were used: gas temperature (nitrogen) $300{ }^{\circ} \mathrm{C}$, drying gas (nitrogen) $7 \mathrm{~L} \mathrm{~min}^{-1}$, nebulizer gas (nitrogen) 10 psig, Vcap $4 \mathrm{kV}$, fragmentor $250 \mathrm{~V}$, skimmer $60 \mathrm{~V}, \mathrm{Vpp}$ (octopole RF) 250 V. The HPLC mobile phases were prepared with HPLC grade solvents. Mobile phase A composition was: $\mathrm{H}_{2} \mathrm{O} 95 \%$, ACN $5 \%$, TFA $0.03 \%$ whilst mobile phase B was ACN $95 \%, \mathrm{H}_{2} \mathrm{O} 5 \%$, TFA $0.03 \%$.
Two samples were analyzed: freshly purified and non-aggregated RIP2fl (phosphorylated RIP2fl) and the same RIP2fl sample supplemented with $0.3 \mathrm{U}$ of lambda protein phosphatase (New England Biolabs) per $\mu$ g of protein (dephosphorylated RIP2fl). The second sample was measured after $1.5 \mathrm{~h}$ of incubation at room temperature. Using a C8 reverse phase micro-column (Zorbax 300SB-C8, $5 \mu \mathrm{m}, 5 \times 0.3 \mathrm{~mm}$, Agilent Technologies) the protein samples were desalted on-line for $3 \mathrm{~min}$ at $100 \mu \mathrm{min}^{-1}$ with $100 \%$ of mobile phase A. After elution at $50 \mu \mathrm{l} \mathrm{min}-1$ with $70 \%$ of mobile phase B, spectra were acquired in the positive ion mode in the $300-3000 \mathrm{~m} / \mathrm{z}$ range. Data were processed with MassHunter software (v. B.02.00, Agilent Technologies) and the number of RIP2fl phosphorylation sites was calculated by comparing the two spectra obtained.

Negative-stain EM. Two different protocols were used to prepare negative-stain grids. For RIP2fl sample, $4 \mu \mathrm{l}$ of sample were applied to the clean side of the carbon on a carbon-mica interface, letting the sample absorb for $20 \mathrm{~s}$. The carbon film was then floated on a drop of $2 \%(\mathrm{w} / \mathrm{v})$ uranyl acetate, picked up with a 400 -mesh copper grid (Electron Microscopy Science) and dried on filter paper (Whatman).

For all the other samples, $6 \mu \mathrm{l}$ of protein solution were applied to glowdischarged carbon coated copper grid (300 mesh, Electron Microscopy Science) and let adsorb for $30 \mathrm{~s}$. Grids were then washed twice in $25 \mu \mathrm{l}$ drop of protein buffer and stained twice for $30 \mathrm{~s}$ with $6 \mu \mathrm{l}$ of $2 \%(\mathrm{w} / \mathrm{v})$ uranyl acetate. Between each step excess of protein/buffer solution/staining was blotted off using a filter paper. Grids were dried on adsorbing paper for at least $5 \mathrm{~min}$ before storage 55,56 .

Negative-stain grids were imaged either with a JEOL 1200-EX II microscope at $100 \mathrm{KV}$ on photographic film or with a Tecnai 12 (FEI) TEM at $120 \mathrm{KV}$ on a Ceta $16 \mathrm{M}$ camera, at a nominal magnification of $16,000 \times$ to $48,000 \times$.

Negative-stain grids prepared to investigate the effect of nucleotides on RIP2 polymerization (see below), were imaged using a Tecnai 12 (FEI) at a nominal magnification of $30,000 x$.

Polymerization of MBP-RIP2fl and RIP2fl. Polymerization of RIP2fl was induced by mixing $20 \mu \mathrm{l}$ of purified tag-free RIP2fl at $0.3 \mathrm{mg} \mathrm{m}^{-1}$ with $5 \mathrm{mM} \mathrm{ATP} \mathrm{(Sigma)}$ and $10 \mathrm{mM} \mathrm{MgCl} 2$. After one hour of incubation at room temperature, sample was visualised by negative-stain $\mathrm{EM}$.

In order to assay the relevance of ATP for RIP2 polymerization, MBP-RIP2fl was purified as RIP2fl, omitting the tag cleavage step. Protein was concentrated until $1 \mathrm{mg} \mathrm{ml}^{-1}$ in final buffer $20 \mathrm{mM}$ Tris $\mathrm{pH} 7.5,50 \mathrm{mM} \mathrm{NaCl}$ and $2 \mathrm{mM} \beta \mathrm{Me}$. Nucleotides (ATP, AMPPCP ADP, AMP, from Sigma) were prepared as follows: nucleotide stocks were dissolved in Milli-Q water at $100 \mathrm{mM}$ and stored at $-80^{\circ} \mathrm{C}$ For each experiment a new aliquot was quickly thawed and diluted twice in $1 \mathrm{M}$ Tris pH 7.5, $100 \mathrm{mM} \mathrm{MgCl} 2$ and successively diluted 10 times in protein sample. MBP-RIP2fl was then incubated with $5 \mathrm{mM}$ ATP, ADP, AMP or AMPPCP (Beta gamma-methylene-adenosine 5'-triphosphate) overnight at room temperature. As controls, we also prepared MBP-RIP2fl and MBP-RIP2fl supplemented with 10 $\mathrm{mM} \mathrm{MgCl} 2$. For each sample one negative-stain grid was prepared and 10 micrographs collected. The entire experiment was repeated twice.

Structure determination of crystMBP-RIP2CARD. Freshly purified crystMBPRIP2CARD was concentrated to $6 \mathrm{mg} \mathrm{ml}^{-1}$ and used immediately for crystallization trials. Initial crystallization conditions were established by testing several commercial screens at the EMBL High Throughput Crystallization Laboratory (Grenoble, France) using a Cartesian robot. The best crystals were obtained at $4^{\circ} \mathrm{C}$ with the sitting drop method from solutions containing $6 \mathrm{mg} \mathrm{ml}^{-1}$ of crystMBPRIP2CARD equilibrated against $0.25 \mathrm{M} \mathrm{NaNO}_{3}$, and 22\% (w/v) PEG 3350. Crystals were transferred to a cryo-protection buffer prepared with mother liquor supplemented with $20 \%(\mathrm{v} / \mathrm{v})$ glycerol, plunged into liquid nitrogen and stored. Diffraction data were collected on ID2957 at the ESRF (Grenoble, France) at a wavelength of $0.976 \AA$ and temperature of $100 \mathrm{~K}$. Data were processed and scaled with $\mathrm{XDS}^{58,59}$. The structure was solved by molecular replacement using PHA$\mathrm{SER}^{60}$ with the NLRP1 CARD domain with a crystallisable MBP at the N-terminus (PDB accession code 4IFP) as search model. There are four fusion proteins per asymmetric unit. Refinement was carried out using cycles of REFMAC5 ${ }^{61}$ and manual rebuilding with COOT $^{62}$. Local NCS constraints and TLS refinement (one set for each MBP and each CARD) were used. Models were validated using Molprobity ${ }^{63}$ from the PHENIX interface. Final statistics are: Ramachandran 95.57\% allowed, $0.44 \%$ outliers; $2.3 \%$ rotamer outliers; Clashscore 1.62; Molprobity score 1.49. Ramachandran outliers are in the MBP molecule structure. Structure figures were produced with $\mathrm{PyMol}^{64}$. Data collection and refinement statistics are in Supplementary Table 1. RMSD calculations were run with Superpose ${ }^{65}$, using the option: Secondary structure matching.

Sample preparation for solid state NMR. RIP2CARD (435-540) cloned into pETM40 (KanaR) was transformed into BL21 star (Thermo Fisher Scientific) for expression. All LB precultures contained $2 \%$ glucose to repress the lac operon. NMR-specific labels (all isotopes from Eurisotop) were introduced by expression at $22^{\circ} \mathrm{C}$ overnight in 1.5 fold M9 medium following the 2-fold concentration method ${ }^{66}$. For uniform ${ }^{13} \mathrm{C}$ and ${ }^{15} \mathrm{~N}$ labelling, $\mathrm{M} 9$ medium was supplemented with $0.7 \mathrm{~g} \mathrm{~L}^{-1}{ }^{15} \mathrm{~N} \mathrm{NH}_{4} \mathrm{Cl}$ and $2.5 \mathrm{~g} \mathrm{~L}^{-113} \mathrm{C}$-glucose. For sparse labelling with $\left[2{ }^{13} \mathrm{C}\right]$ or $\left[1,3-{ }^{13} \mathrm{C}\right]$-glycerol, to measure long range constraints, the protocol previously 
described ${ }^{67}$ was used. Deuteration was achieved by using $\mathrm{D}_{2} \mathrm{O}$ instead of $\mathrm{H}_{2} \mathrm{O}$ for all medium components.

Purification was done in the presence of $2 \mathrm{M}$ urea to prevent aggregation or too early filament formation as reported ${ }^{40}$. Cells from $1.2 \mathrm{~L}$ culture resuspended in $20 \mathrm{mM}$ Tris pH 8, $20 \mathrm{mM} \mathrm{NaCl}, 2 \mathrm{M}$ urea, $2 \mathrm{mM} \beta$-Me, $1 \mathrm{mM}$ Pefabloc (Sigma), $5 \mathrm{mM} \mathrm{MgCl}_{2}$, and $15 \mu \mathrm{L}$ Benzonase (Merck) were disintegrated by high pressure (Microfluidizer LM10, Microfluidics). After centrifugation at 22,000 $\times g$, for $1 \mathrm{~h}$ at $4{ }^{\circ} \mathrm{C}$, the supernatant was filtered $(0.45 \mu \mathrm{m})$ and incubated with about $12 \mathrm{ml} 50 \%$ amylose resin (New England Biolabs) at $15^{\circ} \mathrm{C}$ on a rotator for $3 \mathrm{~h}$. MBPRIP2CARD was eluted with $10 \mathrm{mM}$ maltose in $20 \mathrm{mM}$ Tris $\mathrm{pH} 8,20 \mathrm{mM} \mathrm{NaCl}, 2$ $\mathrm{M}$ urea, $2 \mathrm{mM} \beta$-Me after a washing step. The fusion protein, at about $1 \mathrm{mg} \mathrm{ml}^{-1}$, was cleaved by TEV protease overnight at $22^{\circ} \mathrm{C}$. The next night was used for dialysis against $20 \mathrm{mM}$ Tris $\mathrm{pH} 8,20 \mathrm{mM} \mathrm{NaCl}, 2 \mathrm{mM} \beta$-Me at $8{ }^{\circ} \mathrm{C}$ to remove urea and to begin filament formation lasting a further $48 \mathrm{~h}$ at $20^{\circ} \mathrm{C}$. Filaments were collected by ultracentrifugation at $35000 \times \mathrm{g}$ for $1 \mathrm{~h}$ to produce a pellet for solid state NMR and quality checking by negative-stain EM.

Solid state NMR. The RIP2CARD filaments were packed into the respective rotor by ultracentrifugation at $100000 \times \mathrm{g}$ for $1 \mathrm{~h}$ using a custom-made filling device.

All proton-detected experiments were recorded on a wide-bore $800 \mathrm{MHz}$ spectrometer equipped with a $1.3 \mathrm{~mm}$ triple-resonance MAS probe (Bruker, Karlsruhe, Germany). Typical $\pi / 2$-pulse lengths were $2.5 \mu$ s for ${ }^{1} \mathrm{H}, 5 \mu$ s for ${ }^{13} \mathrm{C}$ and $7 \mu$ s for ${ }^{15} \mathrm{~N}$. The MAS frequency was set to $60 \mathrm{kHz}$ and the sample temperature was kept at approximately $295 \mathrm{~K}$. For the backbone assignment, a standard set of experiments, (H)CANH, (HCO)CA(CO) $\mathrm{NH},(\mathrm{HCA}) \mathrm{CB}(\mathrm{CA}) \mathrm{NH},(\mathrm{HCA}) \mathrm{CB}$ (CACO)NH, (H)CONH and (H)CO(CA)NH, was recorded on ${ }^{2} \mathrm{H},{ }^{13} \mathrm{C},{ }^{15} \mathrm{~N}-$ labeled and $100 \%$ back exchanged RIP2CARD filaments. Water suppression was achieved using the MISSISSIPI sequence and WALTZ-16 was used for ${ }^{13} \mathrm{C}$ and ${ }^{15} \mathrm{~N}$ decoupling during proton detection. The spectra were processed with NMRPipe employing shifted-sinebell and Lorentzian-to-Gaussian apodization functions.

The ${ }^{13} \mathrm{C}-{ }^{13} \mathrm{C}$ DARR correlation spectra were recorded on wide bore 600 and $700 \mathrm{MHz}$ spectrometers equipped with $3.2 \mathrm{~mm}$ triple-resonance MAS probes (Bruker, Karlsruhe, Germany). Typical $\pi / 2$-pulse lengths were $3.1 \mu$ s for ${ }^{1} \mathrm{H}$, and $5 \mu$ s for ${ }^{13} \mathrm{C}$. All 2D spectra were recorded at either 13333 (on the $600 \mathrm{MHz}$ ) or 15 $555 \mathrm{~Hz}(700 \mathrm{MHz}) \mathrm{MAS}$ frequency and a sample temperature of approximately $285 \mathrm{~K}$. Various mixing times, with durations of 10, 50, 150, 300 and $500 \mathrm{~ms}$ were used for the 2-glycerol and 1,3-glycerol-labelled RIP2CARD samples, whereas DARR mixing times of 10 and $50 \mathrm{~ms}$ were used for the uniformly ${ }^{13} \mathrm{C}$-labelled sample. During acquisition and indirect chemical shift evolution a SPINAL64 decoupling scheme with a RF strength of $90 \mathrm{kHz}$ was applied to the proton spins. The data were processed using Topspin version 3.2 (Bruker, Karlsruhe, Germany) applying shifted-sinebell and Lorentzian-to-Gaussian apodization functions. All spectra were recorded using Topspin and were further analyzed with the CCPN Analysis v.2.4.2 package $^{68}$.

Immuno-gold labelling. For immuno-gold labelling experiments, we modified protocol illustrated in $\mathrm{Lu}$, A et al., 2015, as follows. To detect the interaction between NOD2 $\triangle$ LRR and RIP2CARD filaments, purified NOD2 $\triangle$ LRR was mixed with a 50-fold excess of MBP-RIP2CARD and protein polymerization was induced by adding TEV to cleave off the tag. To detect the interaction between NOD2CARDS and RIP2CARD filament, HIS-SUMO-NOD2CARDS ${ }^{s}$ and MBPRIP2CARD were co-purified as described above. Immuno-gold labelling was applied after MBP and HIS-SUMO tags cleavage without further purification.

For immuno-gold labelling, $6 \mu \mathrm{l}$ of sample were applied to glow-discharged carbon coated copper grid (300 mesh, Electron Microscopy Science) and let adsorb for $30 \mathrm{~s}$. Excess sample was blot off on filter paper, and grid was let shaking $10 \mathrm{~min}$ in $30 \mu \mathrm{l}$ drop of PBS $+0.1 \%(\mathrm{w} / \mathrm{v})$ BSA. After removal of excess, grid was transferred to $30 \mu \mathrm{l}$ drops of same solution containing either the rabbit antibody anti-SNAP at dilution 1:2000 (P9310, New England Biolabs), or rabbit anti-NOD2 at dilution 1:1000 (ab197030, Abcam) or no antibody (control sample C2). The specimen was then left in $30 \mu \mathrm{l}$ PBS $+0.1 \%$ (w/v) BSA for 1 min and the same washing procedure was repeated three times. Successively, the grid was floated for $1 \mathrm{~h}$ in a $30 \mu \mathrm{l}$ drop of the same solution containing gold-conjugated goat antibody anti-rabbit at 1:100 dilution (G7277, Sigma). After washing a second time, the grid was stained with $2 \%(\mathrm{w} / \mathrm{v})$ uranyl acetate and images were recorded on a JEOL 1200 at $16,000 x$ or $25,000 x$ magnification.

Grid preparation for cryo-EM and cryo-EM data collection. The sample quality and filament concentration were checked by negative-stain EM prior to cryo-EM grid preparation. Grids for cryo-EM were coated with a thin layer of carbon as follows. A piece of homemade carbon-mica foil was floated for $5 \mathrm{~min}$ on a $30 \mu$ sample on an ice-cooled home-made polyoxymethlene block. A Quantifoil R2.2 grid was glow-discharged for one minute at $25 \mathrm{~mA}$ (PELCO easy glow), applied on top of the floating carbon foil and quickly transferred to a Vitrobot Mark IV (FEI) for vitrification. Vitrobot filter papers (TED PELLA, INC.) were humidified prior to use and the Vitrobot was set to $4{ }^{\circ} \mathrm{C}$ and $100 \%$ humidity. A drop of $3 \mu \mathrm{l}$ water was added to the specimen prior to blotting at a force of -5 for a total time of $45 \mathrm{~s}$. The grid was finally flash-frozen in liquid ethane before storage.
Cryo-EM movies where automatically collected on an FEI Polara electron microscope operated at $300 \mathrm{KV}$. Micrographs were recorded on a K2 direct electron detector operated in counting mode using the software Latitude $\mathrm{S}$, at a nominal magnification of $41270 \times$ (corresponding to $1.21 \AA /$ pixel at the specimen level), with a defocus range of -1.5 to $-3 \mu \mathrm{m}$. 720 movies of 40 frames were collected with a total dose of 50 electrons $\AA^{-2}$ (Supplementary Table 3).

Image processing, symmetry determination and structure refinement. After selection according to ice quality, half of the movies collected were actually used for data processing. The frames 2 to 16, corresponding to a total dose of 20 electrons $\AA^{-2}$, were motion-corrected and dose-weighted using MotionCor $2^{69}$. The defocus estimation was performed with CTFFIND/CTFTILT ${ }^{70}$.

Initially, 4125 sections of filaments (total length $97 \mu \mathrm{m}$ ) were manually boxed using the e2helixboxer module of EMAN2 ${ }^{71}$, while avoiding to include the filament-ends where the hetero-CARD complex could be situated. This manually picked data set was analysed with RELION ${ }^{72}$, to obtain $2 \mathrm{D}$ class-averages used both as templates for automatic picking, and for initial symmetry estimation from their power spectrum. To this end, the filaments were segmented with an inter-box distance of $25 \AA$ into 23742 segments of size $420^{*} 420$ pixels, which were used for $2 \mathrm{D}$ classification, asking for thirty classes. Three significantly populated $2 \mathrm{D}$ classaverages were obtained, amongst which two correspond to straight segments, and show a clear repetitive pattern along the helical axis (Fig. 6b). The individual power spectra of these two 2D class-averages were nearly identical, and showed layer lines up to $\sim 1 / 4.9 \AA^{-1}$ (Fig. 6 c), which enabled a first estimation of the helical parameters as follows (Supplementary Fig. 8a). The layer lines are spaced regularly at multiples of $\sim 1 / 155.8 \AA^{-1}$, giving an estimation of the repeat $c$. A meridional layer line $\mathrm{l}=32$ (Bessel order $\mathrm{n}=0$ ), with a height of $1 / 4.87 \AA^{-1}$ indicated the axial rise between subunits. A strong layer line $\mathrm{l}=9$ with a first intensity maximum near the meridian $(\mathrm{n}=1)$, at $1 / 17.3 \AA^{-1}$, was attributed to the pitch $\mathrm{P}$, as observed for other CARD domains filamentous assemblies (Supplementary Table 4 ). Therefore, the structure repeats after $\mathrm{u}=32$ subunits $(32 * 4.87=155.8)$, in $\mathrm{t}=9$ turns, resulting in a number of units per turn $(\mathrm{u} / \mathrm{t})$ of $\sim 3.56$.

The two straight $2 \mathrm{D}$ class-averages were subsequently used as templates for automatic picking of helical segments in RELION, using a maximum curvature parameter $\kappa=0.4$, and a minimum filament length of $800 \AA$. The coordinates of filament extremities were converted into EMAN2 format, while shortening them by $180 \AA$ at each extremity, to avoid including the hetero-CARD complex. This resulted in 4443 filament sections for a total length of $260 \mu \mathrm{m}$.

All subsequent processing steps were performed in the helical reconstruction software package SPRING ${ }^{73}$. An initial model was obtained by $3 \mathrm{D}$ refinement from the manually picked data set and the symmetry parameters as estimated from the $2 \mathrm{D}$ class average power spectrum indexing, with low-pass filtering to $20 \AA$. The auto-picked filament coordinates were used to extract 37043 segments using a segment length of $400 \AA$ and segment step size of $70 \AA$. The symmetry parameters were further refined with the module Segrefine3Dgrid, by defining a $11^{\star} 11$ grid spanning between 17.2-17.4 $\AA$ (step $0.02 \AA$ ) for the pitch and between 3.4-3.6 (step 0.02 ) for the number of units per turn. The maximum of the amplitude correlation between experimental and reprojection power spectra was found at a pitch of 17.26 $\AA$, 3.56 units per turn (Supplementary Fig. 8b), corresponding to a left-handed helix with $-101.124^{\circ}$ helical twist and $4.848 \AA$ axial rise. Using the refined symmetry parameters, we performed a high-resolution structure refinement using the autopicked segments with a strict segment selection during refinement, based on geometrical restraints ${ }^{73,74}$ namely filament straightness $(70 \%$ of straightest filaments kept) and forward $\mathrm{x}$-shift difference (limited to $5 \AA$ ). This resulted in a final reconstruction including 9661 segments (corresponding to 135,254 asymmetric units after symmetrisation) at a resolution of $3.94 \AA$ (FSC between half data set maps, cut off 0.143, Supplementary Fig. 8c). For visual display and the model building, the EM map was filtered to $3.9 \AA$ and sharpened using a B-factor of $-200 \AA^{2}$.

Visualization of the resulting map and initial rigid body fitting of the crystallographic structure was done using Chimera ${ }^{75}$. Atomic model refinement was done using PHENIX real space refinement ${ }^{11}$ and manual adjustment with $\operatorname{Coot}^{62}$. The crystallographic model was used to assign side chains for the residues without clear EM density map. Structure validation was done with Molprobity ${ }^{63}$. Figures were prepared with PyMOL ${ }^{64}$ and Chimera. Software used in this project was installed and configured by SBGrid ${ }^{76}$. Data collection, image processing and refinement statistics are reported in Supplementary Table 3 and Supplementary Fig. 8 d.

\section{Production of RIP2CARD mutants and Western blots. RIP2CARD mutants} were produced by PCR mutagenesis of the HIS-MBP-RIP2CARD construct, using the oligos and their complements as reported in Supplementary Table 5. Expression was performed as described above.

For co-production of RIP2CARD mutants with NOD2CARDS, $0.250 \mathrm{ml}$ $(\sim 2.5 \mathrm{~g}$ of bacterial pellet) of HIS-MBP- RIP2CARD mutants culture was combined with $100 \mathrm{ml}$ of NOD2CARDS ${ }^{s}(\sim 9 \mathrm{~g}$ of bacterial pellet) and resuspended in $50 \mathrm{ml}$ of lysis buffer $(20 \mathrm{mM}$ Tris $\mathrm{pH} 8,50 \mathrm{mM} \mathrm{NaCl}, 1 \mathrm{mM}$ TCEP and Complete protease inhibitor cocktail (Roche)). The sample was then treated as described for NOD2CARDS ${ }^{s}$-RIP2CARD filament sample. After elution from the amylose resin, complex formation was checked on SDS-PAGE, and presence of 
HIS-SUMO-NOD2CARDS ${ }^{s}$ confirmed by western blot (WB). For WB, $8 \mu \mathrm{g}$ of total protein was loaded on either a 17\% SDS-PAGE gel (Fig. 5b) or stain-free $4-12 \%$ gradient SDS-PAGE gel (Bio-Rad) (Fig. 8b). Rabbit anti-SNAP at 1:2000 dilution (P9310,New England Biolabs) was used for detection of NOD2CARDS . For WB revelation, a goat anti-rabbit secondary antibody linked to Alkaline Phosphatase was used at 1:30000 dilution (A3687, Sigma). Uncropped blots are shown in Supplementary Fig. 13.

Filament polymerization was stimulated by adding proteases for $4 \mathrm{~h}$ at room temperature, at a protein concentration of $2.5 \mathrm{mg} \mathrm{ml}^{-1}$. Negative-stain EM was used to check the polymerization state of each sample.

Experiments were repeated twice for constructs that showed impaired RIP2CARD polymerization.

Mammalian cell culture and plasmids. A HEK293T cell line (from the laboratory of W.Filipowicz) was used. The cell line has not been authenticated but has been tested and shown to be free of mycoplasma. Cells were maintained in DMEM medium (Lonza) supplemented with $10 \%(\mathrm{v} / \mathrm{v})$ fetal bovine serum (FBS) and nonessential amino acids (Gibco), at $37^{\circ} \mathrm{C}$ and $5 \% \mathrm{CO}_{2}$. Constructs for in cell based assays, were generated from pcDNA3 plasmids encoding for human RIP2 $\mathrm{fl}^{50}$. The original pcDNA3-RIP2(10-540) was modified into pcDNA-HA-RIP2(1-540), using the oligos reported in Supplementary Table 5, which resulted in a construct encoding for full-length RIP2 with an HA tag at the N-terminus and a GSAGSA linker between tag and protein. Single amino acids mutants were obtained by sitedirected PCR mutagenesis of pcDNA3-HA-RIP2(1-540), using the oligos listed in Supplementary Table 5 .

In cell reported luciferase assay and Western blots. HEK293T cells were seeded in 12-well plates $24 \mathrm{~h}$ prior transfection. Transfection was performed with LipoD293 transfection reagent (SignaGen). Each well was transfected with $5 \mathrm{ng}$ pcDNA3 plasmid encoding for pcDNA3-HA-RIP2 or corresponding mutant, $1 \mathrm{ng}$ of pCDNA3, $500 \mathrm{ng}$ of Luc-NF- $\mathrm{KB}^{50}$, encoding a firefly luciferase reporter gene under NF-kB promoter and $50 \mathrm{ng}$ of pRenilla-TK plasmid. As negative control, transfection mix with pcDNA3-HA-RIP2 replaced with empty vector was used. pRenilla-TK was used to correct for the transfection efficiencies. Each transfection mixture was prepared in double and completed with either the NOD2 activator MDP or its corresponding control cMDP (Invivogen). Cells were lysed $20 \mathrm{~h}$ after transfection in $250 \mu \mathrm{l}$ of lysis buffer, accordingly to the manufacturer protocol Dual Luciferase assay (Promega). Sample was then used for luciferases activities measurement and for western blots (WB). Firefly and Renilla luciferase activities were measured using a Clariostar microplate reader using the double injector system (BMG Germany). $10 \mu \mathrm{l}$ per sample were dispensed in a luciferase specific 96-wells plate (Thermo Scientific). $25 \mu \mathrm{l}$ of Firefly developing solution (LucII) were firstly injected, the plate was stirred at $500 \mathrm{rpm}$ for $15 \mathrm{~s}$, and 12 measurements performed. $25 \mu \mathrm{l}$ of Stop and Glow solution were then dispensed. The plate was stirred again and the same number of measurements were recorded. Each experiment was repeated three times (biological replicate) and each time, three transfections per condition were performed (technical replicate), for a total of nine experiments per construct.

For WB, $10 \mu \mathrm{g}$ of total protein were loaded on a stain-free $4-12 \%$ gradient SDSPAGE gel (Bio-Rad). Mouse anti-HA (26183, Thermo Fisher) at 1:2000 dilution was used for detection of transfected HA-RIP2 and its corresponding mutants, whilst anti $\beta$-actin antibody at 1:2000 dilution was employed for normalization of total protein amount (ab8227, Abcam). For WB revelation secondary antibodies linked to fluorophores were used 1:2000 dilution, goat anti-mouse and goat antirabbit linked to Alexa 532 and Alexa 633 respectively (A11002 and A21070 respectively, Thermo Fisher). A representative WB was run for each biological replicate. Uncropped blots are shown in Supplementary Fig. 14 and 15.

Statistical analysis. Statistical analysis on the luciferase reporter assay was performed using Rstudio ${ }^{77,78}$ with the aim of quantitatively finding evidence of significant differences between the two experiments, namely with NOD2 ligand MDP and the control cMDP. Their mean response was assessed using a parametric method; hence each of the samples was evaluated using a Shapiro Wilk test for normality. The null hypothesis assumed normality, and all samples failed to reject this hypothesis test showing at $95 \%$ of significance that they were all normally distributed. Moreover, samples were assumed to have homogenous variances to satisfy the requirements of the parametric tests. The Student $t$-test on two independent samples (two-sided) was conducted for each pair of conditions. Firstly, wild-type RIP2 and vector showed, without ambiguity according to p-value, that their respective means were different. Then, wild-type RIP2 was compared to the mean of each mutant. Except for wild-type RIP2 vs mutant Q458A, Q458K, N512A and $\mathrm{N} 512 \mathrm{~K}$ where no significant differences from their means was observed, all other mutants rejected more or less strongly the null hypothesis. In other words, their mean responses were significantly different from that of wild-type RIP2. Finally, the Student $t$-test comparison of two independent samples were conducted on "MDP" and "cMDP" version of each mutants, including both vector and wildtype RIP2. All conditions rejected the null hypothesis; leading to the conclusion that their means were different at $95 \%$ significance. Once quantitative evidence about differences from MDP and cMDP experiment was confirmed, a boxplot was generated to visualise the results (Fig. 9).

\section{Data availability}

Coordinates and structure factors for crystMBP-RIP2CARD are deposited in the PDB with accession code 6GFJ [https://www.rcsb.org/structure/6GFJ]. Coordinates for the RIP2CARD filament have accession code 6GGS [https://www.rcsb.org/structure/6GGS]. The cryoEM map has accession code EMD-4399. The solid-state NMR data is deposited in the BMRB database with accession number 27555 (this data can be retrieved using the restart ID 2018-07-16.deposit.bmrb.wisc.edu.80.13422729 at http:// deposit.bmrb.wisc.edu/bmrb-adit/access.html).

Received: 25 April 2018 Accepted: 6 September 2018 Published online: 02 October 2018

\section{References}

1. Takeuchi, O. \& Akira, S. Pattern recognition receptors and inflammation. Cell 140, 805-820 (2010).

2. Ogura, Y. et al. Nod2, a Nod1/Apaf-1 family member that is restricted to monocytes and activates NF-kappaB. J. Biol. Chem. 276, 4812-4818 (2001).

3. Girardin, S. E. et al. Nod2 is a general sensor of peptidoglycan through muramyl dipeptide (MDP) detection. J. Biol. Chem. 278, 8869-8872 (2003).

4. Inohara, N. et al. Host recognition of bacterial muramyl dipeptide mediated through NOD2. Implications for Crohn's disease. J. Biol. Chem. 278, 5509-5512 (2003)

5. Magalhaes, J. G. et al. Essential role of Rip2 in the modulation of innate and adaptive immunity triggered by Nod1 and Nod2 ligands. Eur. J. Immunol. 41, 1445-1455 (2011).

6. Ting, J. P. et al. The NLR gene family: a standard nomenclature. Immunity $\mathbf{2 8}$, 285-287 (2008)

7. Maekawa, S., Ohto, U., Shibata, T., Miyake, K. \& Shimizu, T. Crystal structure of NOD2 and its implications in human disease. Nat. Commun. 7, 11813 (2016).

8. Humphries, F., Yang, S., Wang, B. \& Moynagh, P. N. RIP kinases: key decision makers in cell death and innate immunity. Cell Death Differ. 22, 225-236 (2015).

9. Boyle, J. P., Parkhouse, R. \& Monie, T. P. Insights into the molecular basis of the NOD2 signalling pathway. Open biology 4, https://doi.org/10.1098/ rsob.140178 (2014).

10. Philpott, D. J., Sorbara, M. T., Robertson, S. J., Croitoru, K. \& Girardin, S. E. NOD proteins: regulators of inflammation in health and disease. Nat. Rev. Immunol. 14, 9-23 (2014)

11. Rubino, S. J., Selvanantham, T., Girardin, S. E. \& Philpott, D. J. Nod-like receptors in the control of intestinal inflammation. Curr. Opin. Immunol. 24, 398-404 (2012).

12. Adams, P. D. et al. PHENIX: a comprehensive Python-based system for macromolecular structure solution. Acta Crystallographica D Biological Crystallogr. 66, (213-221 (2010).

13. Fridh, V. \& Rittinger, K. The tandem CARDs of NOD2: intramolecular interactions and recognition of RIP2. PLoS ONE 7, e34375 (2012).

14. Wagner, R. N., Proell, M., Kufer, T. A. \& Schwarzenbacher, R. Evaluation of Nod-like receptor (NLR) effector domain interactions. PLoS ONE 4, e4931 (2009).

15. Damgaard, R. B. et al. The ubiquitin ligase XIAP recruits LUBAC for NOD2 signaling in inflammation and innate immunity. Mol. Cell 46, 746-758 (2012).

16. Bertrand, M. J. et al. Cellular inhibitors of apoptosis cIAP1 and cIAP2 are required for innate immunity signaling by the pattern recognition receptors NOD1 and NOD2. Immunity 30, 789-801 (2009).

17. Goncharov, T. et al. Disruption of XIAP-RIP2 association blocks NOD2mediated inflammatory signaling. Mol. Cell 69, 551-565 (2018). e557.

18. Windheim, M., Lang, C., Peggie, M., Plater, L. A. \& Cohen, P. Molecular mechanisms involved in the regulation of cytokine production by muramyl dipeptide. Biochem. J. 404, 179-190 (2007).

19. Homer, C. R. et al. A dual role for receptor-interacting protein kinase 2 (RIP2) kinase activity in nucleotide-binding oligomerization domain 2 (NOD2)dependent autophagy. J. Biol. Chem. 287, 25565-25576 (2012).

20. Hugot, J. P. CARD15/NOD2 mutations in Crohn's disease. Ann. N. Y. Acad. Sci. 1072, 9-18 (2006).

21. Kaplan, G. G. The global burden of IBD: from 2015 to 2025. Nature reviews. Gastroenterol. \& Hepatol. 12, 720-727 (2015).

22. Liu, J. Z. \& Anderson, C. A. Genetic studies of Crohn's disease: past, present and future. Best. Pract. \& Res. Clin. Gastroenterol. 28, 373-386 (2014).

23. Wehkamp, J. \& Stange, E. F. Paneth's disease. J. Crohn's \& Colitis 4, 523-531 (2010).

24. Miceli-Richard, C. et al. CARD15 mutations in Blau syndrome. Nat. Genet. 29, 19-20 (2001). 
25. Caso, F. et al. Autoinflammatory granulomatous diseases: from Blau syndrome and early-onset sarcoidosis to NOD2-mediated disease and Crohn's disease. RMD Open 1, e000097 (2015).

26. Henckaerts, L. \& Vermeire, S. NOD2/CARD15 disease associations other than Crohn's disease. Inflamm. Bowel Dis. 13, 235-241 (2007).

27. Kanazawa, N. et al. Early-onset sarcoidosis and CARD15 mutations with constitutive nuclear factor-kappaB activation: common genetic etiology with Blau syndrome. Blood 105, 1195-1197 (2005).

28. Xie, T. et al. Structural insights into RIP3-mediated necroptotic signaling. Cell Rep. 5, 70-78 (2013)

29. Wu, H. \& Fuxreiter, M. The structure and dynamics of higher-order assemblies: amyloids, signalosomes, and granules. Cell 165, 1055-1066 (2016).

30. Hou, F. et al. MAVS forms functional prion-like aggregates to activate and propagate antiviral innate immune response. Cell 146, 448-461 (2011)

31. Wu, B. et al. Molecular imprinting as a signal-activation mechanism of the viral RNA sensor RIG-I. Mol. Cell 55, 511-523 (2014).

32. $\mathrm{Xu}, \mathrm{H}$. et al. Structural basis for the prion-like MAVS filaments in antiviral innate immunity. eLife 3, e01489 (2014).

33. $\mathrm{Lu}, \mathrm{A}$. et al. Unified polymerization mechanism for the assembly of ASCdependent inflammasomes. Cell 156, 1193-1206 (2014).

34. Lu, A. et al. Molecular basis of caspase-1 polymerization and its inhibition by a new capping mechanism. Nat. Struct. Mol. Biol. 23, 416-425 (2016).

35. David, L. et al. Assembly mechanism of the CARMA1-BCL10-MALT1TRAF6 signalosome. Proc. Natl Acad. Sci. USA https://doi.org/10.1073/ pnas.1721967115 (2018).

36. Pellegrini, E., Signor, L., Singh, S., Boeri Erba, E. \& Cusack, S. Structures of the inactive and active states of RIP2 kinase inform on the mechanism of activation. PLoS ONE 12, e0177161 (2017).

37. Jang, T. H. et al. Structural study of the RIPoptosome core reveals a helical assembly for kinase recruitment. Biochemistry 53, 5424-5431 (2014).

38. Waugh, D. S. Crystal structures of MBP fusion proteins. Protein Sci.: a Publ. Protein Soc. 25, 559-571 (2016).

39. Jin, T., Curry, J., Smith, P., Jiang, J. \& Xiao, T. S. Structure of the NLRP1 caspase recruitment domain suggests potential mechanisms for its association with procaspase-1. Proteins 81, 1266-1270 (2013).

40. Lin, Z. et al. Structural basis of death domain signaling in the p75 neurotrophin receptor. elife 4, e11692 (2015).

41. Dephoure, N. et al. A quantitative atlas of mitotic phosphorylation. Proc. . Natl Acad. Sci. USA 105, 10762-10767 (2008).

42. Zhou, $\mathrm{H}$. et al. Toward a comprehensive characterization of a human cancer cell phosphoproteome. J. Proteome Res. 12, 260-271 (2013)

43. Daub, $H$. et al. Kinase-selective enrichment enables quantitative phosphoproteomics of the kinome across the cell cycle. Mol. Cell 31, 438-448 (2008).

44. Barbet-Massin, E. et al. Rapid proton-detected NMR assignment for proteins with fast magic angle spinning. J. Am. Chem. Soc. 136, 12489-12497 (2014).

45. Castellani, F. et al. Structure of a protein determined by solid-state magicangle-spinning NMR spectroscopy. Nature 420, 98-102 (2002).

46. Higman, V. A. et al. Assigning large proteins in the solid state: a MAS NMR resonance assignment strategy using selectively and extensively 13C-labelled proteins. J. Biomol. NMR 44, 245-260 (2009).

47. Ferrao, R. \& Wu, H. Helical assembly in the death domain (DD) superfamily. Curr. Opin. Struct. Biol. 22, 241-247 (2012).

48. Biasini, M. et al. SWISS-MODEL: modelling protein tertiary and quaternary structure using evolutionary information. Nucleic Acids Res. 42, W252-W258 (2014).

49. Jang, T. H. et al. Crystal structure of caspase recruiting domain (CARD) of apoptosis repressor with CARD (ARC) and its implication in inhibition of apoptosis. Sci. Rep. 5, 9847 (2015).

50. Manon, F., Favier, A., Nunez, G., Simorre, J. P. \& Cusack, S. Solution structure of NOD1 CARD and mutational analysis of its interaction with the CARD of downstream kinase RICK. J. Mol. Biol. 365, 160-174 (2007).

51. Nanson, J. D., Rahaman, M. H., Ve, T. \& Kobe, B. Regulation of signaling by cooperative assembly formation in mammalian innate immunity signalosomes by molecular mimics. Semin. Cell Dev. Biol. https://doi.org/10.1016/j. semcdb.2018.05.002 (2018)

52. Dorsch, M. et al. Identification of a regulatory autophosphorylation site in the serine-threonine kinase RIP2. Cell Signal. 18, 2223-2229 (2006).

53. Li, J. et al. The RIP1/RIP3 necrosome forms a functional amyloid signaling complex required for programmed necrosis. Cell 150, 339-350 (2012).

54. Tigno-Aranjuez, J. T., Asara, J. M. \& Abbott, D. W. Inhibition of RIP2's tyrosine kinase activity limits NOD2-driven cytokine responses. Genes Dev. 24, 2666-2677 (2010).

55. Booth, D. S., Avila-Sakar, A. \& Cheng, Y. Visualizing proteins and macromolecular complexes by negative stain EM: from grid preparation to image acquisition. Journal of visualized experiments: JoVE, https://doi.org/ $10.3791 / 3227$ (2011)
56. Ohi, M., Li, Y., Cheng, Y. \& Walz, T. Negative staining and image classification - powerful tools in modern electron microscopy. Biol. Proced. Online 6, 23-34 (2004).

57. Flot, D. et al. The ID23-2 structural biology microfocus beamline at the ESRF J. Synchrotron Radiat. 17, 107-118 (2010).

58. Kabsch, W. Xds.Acta Crystallogr. D Biol. Crystallogr. 66, 125-132 (2010).

59. Karplus, P. A. \& Diederichs, K. Linking crystallographic model and data quality. Science 336, 1030-1033 (2012).

60. McCoy, A. J. et al. Phaser crystallographic software. J. Appl. Crystallogr. 40 , 658-674 (2007).

61. Murshudov, G. N., Vagin, A. A. \& Dodson, E. J. Refinement of macromolecular structures by the maximum-likelihood method. Acta Crystallogr. D. Biol. Crystallogr. 53, 240-255 (1997)

62. Emsley, P. \& Cowtan, K. Coot: model-building tools for molecular graphics. Acta crystallographica. Section D. Biol. Crystallogr. 60, 2126-2132 (2004)

63. Chen, V. B. et al. MolProbity: all-atom structure validation for macromolecular crystallography. Acta Crystallogr. D Biol. Crystallogr. 66 12-21 (2010).

64. DeLano, W. L. PyMOL Molecular Graphics System http://www.pymol. sourceforge.net (2002)

65. Krissinel, E. \& Henrick, K. Secondary-structure matching (SSM), a new tool for fast protein structure alignment in three dimensions. Acta Crystallogr. D. Biol. Crystallogr. 60, 2256-2268 (2004).

66. Marley, J., Lu, M. \& Bracken, C. A method for efficient isotopic labeling of recombinant proteins. J. Biomol. NMR 20, 71-75 (2001)

67. LeMaster, D. M. \& Kushlan, D. M. Dynamical Mapping of E. coli Thioredoxin via 13C NMR Relaxation Analysis. J. Am. Chem. Soc. 118, 9255-9264 (1996)

68. Stevens, T. J. et al. A software framework for analysing solid-state MAS NMR data. J. Biomol. NMR 51, 437-447 (2011).

69. Zheng, S. Q. et al. MotionCor2: anisotropic correction of beam-induced motion for improved cryo-electron microscopy. Nat. Methods 14, 331-332 (2017).

70. Mindell, J. A. \& Grigorieff, N. Accurate determination of local defocus and specimen tilt in electron microscopy. J. Struct. Biol. 142, 334-347 (2003).

71. Tang, G. et al. EMAN2: an extensible image processing suite for electron microscopy. J. Struct. Biol. 157, 38-46 (2007).

72. Scheres, S. H. RELION: implementation of a Bayesian approach to cryo-EM structure determination. J. Struct. Biol. 180, 519-530 (2012).

73. Desfosses, A., Ciuffa, R., Gutsche, I. \& Sachse, C. SPRING - an image processing package for single-particle based helical reconstruction from electron cryomicrographs. J. Struct. Biol. 185, 15-26 (2014).

74. Sachse, C. et al. High-resolution electron microscopy of helical specimens: a fresh look at tobacco mosaic virus. J. Mol. Biol. 371, 812-835 (2007).

75. Pettersen, E. F. et al. UCSF Chimera--a visualization system for exploratory research and analysis. J. Comput. Chem. 25, 1605-1612 (2004).

76. Morin, A. et al. Collaboration gets the most out of software. eLife 2, e01456 (2013).

77. The R-core team. The R Project for Statistical Computing http://www.r-project. org/. (2017)

78. Wickman, H. ggplot2:Elegant Graphics for data analysis (Springer-Verlag, New York, 2009).

79. Wishart, D. S. \& Sykes, B. D. The $13 \mathrm{C}$ chemical-shift index: a simple method for the identification of protein secondary structure using $13 \mathrm{C}$ chemical-shift data. J. Biomol. NMR 4, 171-180 (1994)

80. Wishart, D. S. \& Sykes, B. D. Chemical shifts as a tool for structure determination. Methods Enzymol. 239, 363-392 (1994).

\section{Acknowledgements}

We thank the staff of the ESRF-EMBL Joint Structural Biology Group for access to and help on ESRF beamlines. We acknowledge Alice Aubert for support in using the Eukaryotic Expression Facility and the staff of the high-throughput crystallization facility, both at EMBL Grenoble. We are grateful to Taiana M. Oliveira for help with early negative-stain EM experiments and Patricia Resa Infante for help with establishing the in cell assays. We thank the EM platform at the IBS Grenoble, especially Emmanuelle Neumann for technical support and access. This platform is partly supported by the Rhône-Alpes Region, the Fondation pour la Recherche Médicale (FRM), the FEDER fund and the GIS Infrastructures en Biologie Santé et Agronomie (IBISA). We thank Anne Diehl for technical assistance in NMR sample preparation. We acknowledge the assistance of Martina Ringling from the team of Dmytro Puchkov and Matthias Hiller in the NMR facility at the Leibniz-Institut für Molekulare Pharmakologie in Berlin. We used the platforms of the Grenoble Instruct-ERIC Center (ISBG: UMS 3518 CNRS-CEAUGA-EMBL) with support from FRISBI (ANR-10-INSB-05-02) and GRAL (ANR-10LABX-49-01) within the Grenoble Partnership for Structural Biology (PSB). A.D. was supported by an FRM grant (ARF20160936266). 


\section{Author contributions}

E.P. and S.C. conceived and directed the project. E.P performed biochemical and biophysical experiments alone or together with I.G. and H.M. (negative-stain EM on RIP2fl, Fig. 1 and Supplementary Fig. 1), G.K. (crystallisation of RIP2CARD, Fig. 3 and Supplementary Fig. 3; Purification of NOD2 $\triangle$ LRR, Fig. 4), S.G. (Co-purification and immuno-gold labelling experiments, Figs 4, 5), M.H. (preparation and screening of cryo-EM grids, Fig. 6 and Supplementary Fig. 7) and W.M.S (in vitro analysis of RIP2CARD mutants, Fig. 8 and Supplementary Fig. 11). K.R. purified protein for NMR experiments. A.W. and H.O measured and analysed solid-state NMR data. E.P., H.M. and C.S. performed initial EM data processing. G.S. did the Polara cryo-EM data collection. E.P. and A.D. performed the helical reconstruction. E.P. and S.C. performed the crystallographic structure determination. P.M. performed statistical analysis. L.S. performed mass spectrometry experiments. E.P. and S.C. wrote the manuscript with input from all authors.

\section{Additional information}

Supplementary Information accompanies this paper at https://doi.org/10.1038/s41467018-06451-3.

Competing interests: The authors declare no competing interests.

Reprints and permission information is available online at http://npg.nature.com/ reprintsandpermissions/
Publisher's note: Springer Nature remains neutral with regard to jurisdictional claims in published maps and institutional affiliations.

\section{(c) (i)}

Open Access This article is licensed under a Creative Commons Attribution 4.0 International License, which permits use, sharing, adaptation, distribution and reproduction in any medium or format, as long as you give appropriate credit to the original author(s) and the source, provide a link to the Creative Commons license, and indicate if changes were made. The images or other third party material in this article are included in the article's Creative Commons license, unless indicated otherwise in a credit line to the material. If material is not included in the article's Creative Commons license and your intended use is not permitted by statutory regulation or exceeds the permitted use, you will need to obtain permission directly from the copyright holder. To view a copy of this license, visit http://creativecommons.org/ licenses/by/4.0/.

(C) The Author(s) 2018 